\title{
POINCARÉ-DULAC NORMAL FORM REDUCTION FOR UNCONDITIONAL WELL-POSEDNESS OF THE PERIODIC CUBIC NLS
}

\author{
ZIHUA GUO, SOONSIK KWON, AND TADAHIRO OH
}

\begin{abstract}
We implement an infinite iteration scheme of Poincaré-Dulac normal form reductions to establish an energy estimate on the one-dimensional cubic nonlinear Schrödinger equation (NLS) in $C_{t} L^{2}(\mathbb{T})$, without using any auxiliary function space. This allows us to construct weak solutions of NLS in $C_{t} L^{2}(\mathbb{T})$ with initial data in $L^{2}(\mathbb{T})$ as limits of classical solutions. As a consequence of our construction, we also prove unconditional well-posedness of NLS in $H^{s}(\mathbb{T})$ for $s \geq \frac{1}{6}$.
\end{abstract}

\section{Contents}

1. Introduction

1.1. Nonlinear Schrödinger equation

1.2. Poincaré-Dulac normal form reduction: formal argument

2. Poincaré-Dulac normal form reduction, Part 1: basic setup

3. Poincaré-Dulac normal form reduction, Part 2: infinite iteration

3.1. Notations: index by trees

3.2. Example: second and third generations

3.3. General step: $J$ th generation

4. Existence of weak solutions

5. Unconditional uniqueness in $C_{t} H^{s}, s \geq \frac{1}{6}$

References

\section{INTRODUCTION}

1.1. Nonlinear Schrödinger equation. We consider the Cauchy problem for the cubic nonlinear Schrödinger equation (NLS) on the one-dimensional torus $\mathbb{T}=\mathbb{R} / \mathbb{Z}$ :

$$
\left\{\begin{array}{l}
i u_{t}-u_{x x}= \pm u|u|^{2} \\
\left.u\right|_{t=0}=u_{0},
\end{array} \quad(x, t) \in \mathbb{T} \times \mathbb{R},\right.
$$

where $u$ is a complex-valued function. In this paper, we study unconditional well-posedness of (1.1). One-dimensional cubic NLS is known to be completely integrable. However, our

2000 Mathematics Subject Classification. 35Q55.

Key words and phrases. NLS; normal form; well-posedness; uniqueness.

Z.G. is supported by the National Science Foundation under agreement No. DMS-0635607 and The S. S. Chern Fund. Any opinions, findings and conclusions or recommendations expressed in this material are those of the authors and do not necessarily reflect the views of the National Science Foundation or The S. S. Chern Fund.

S.K. is supported in part by NRF grant 2010-0024017. 
argument does not use such integrability structure of (1.1) in an explicit manner. Moreover, due to the local-in-time nature of our argument, it does not matter whether the equation is defocusing (with - sign) or focusing (with + sign.) Hence, we simply assume that it is defocusing in the following.

In [3], Bourgain introduced a new weighted space time Sobolev space $X^{s, b}$, whose norm is given by

$$
\|u\|_{X^{s, b}(\mathbb{T} \times \mathbb{R})}=\left\|\left\langle\partial_{x}\right\rangle^{s}\left\langle i \partial_{t}-\partial_{x}^{2}\right\rangle^{b}(u)\right\|_{L^{2}(\mathbb{T} \times \mathbb{R})},
$$

where $\langle\cdot\rangle=1+|\cdot|$. After establishing the periodic $L^{4}$-Strichartz estimate

$$
\|u\|_{L_{x, t}^{4}} \lesssim\|u\|_{X^{0, \frac{3}{8}}}
$$

Bourgain proved that (1.1) is locally well-posed in $L^{2}(\mathbb{T})$. Thanks to the $L^{2}$-conservation law, this immediately implied global well-posedness of $(1.1)$ in $L^{2}(\mathbb{T})$. This well-posedness result is known to be sharp in view of the ill-posedness results of (1.1) in $H^{s}(\mathbb{T}), s<0$, by Burq-Gérard-Tzvetkov [6], Christ-Colliander-Tao [9, 10], and Molinet [18]. Recently, there have been several studies on constructing solutions of (the renormalized version of) (1.1) in larger spaces than $L^{2}(\mathbb{T})$. See Christ [7] and Colliander-Oh [12].

Our main goal in this paper is twofold.

(a) We construct weak solutions of (1.1) with $u_{0} \in L^{2}(\mathbb{T})$, by directly establishing an energy estimate in $C\left([0, T] ; L^{2}\right)$ without using any auxiliary function space.

(b) We establish a uniqueness statement of solutions of (1.1). For this part, we assume sufficient regularity on solutions. In particular, we assume that a solution $u$ is in $C\left([0, T] ; H^{s}\right)$ for some $s \geq \frac{1}{6}$.

First, let us discuss what we mean by solutions in $C\left([0, T] ; L^{2}\right)$. For this purpose, we use the following notions from Christ [7, 8].

Definition 1.1. A sequence of Fourier cutoff operators is a sequence of Fourier multiplier operators $\left\{T_{N}\right\}_{N \in \mathbb{N}}$ on $\mathcal{D}^{\prime}(\mathbb{T})$ with multipliers $m_{N}: \mathbb{Z} \rightarrow \mathbb{C}$ such that (i) $m_{N}$ has a compact support on $\mathbb{Z}$ for each $N \in \mathbb{N}$, (ii) $m_{N}$ is uniformly bounded, and (iii) $m_{N}$ converges pointwise to 1 , i.e. $\lim _{N \rightarrow \infty} m_{N}(n)=1$ for any $n \in \mathbb{Z}$.

The following definition is in particular important in making sense of the nonlinearity $\mathcal{N}(u):=u|u|^{2}$, when a function $u$ is merely in $C\left([0, T]: L^{2}(\mathbb{T})\right)$.

Definition 1.2. Let $u \in C\left([0, T] ; L^{2}(\mathbb{T})\right)$. We say that $\mathcal{N}(u)$ exists and is equal to a distribution $w \in \mathcal{D}^{\prime}(\mathbb{T} \times(0, T))$ if for every sequence $\left\{T_{N}\right\}_{N \in \mathbb{N}}$ of (spatial) Fourier cutoff operators, we have

$$
\lim _{N \rightarrow \infty} \mathcal{N}\left(T_{N} u\right)=w
$$

in the sense of distributions on $\mathbb{T} \times(0, T)$.

Definition 1.3. We say that $u \in C\left([0, T] ; H^{s}(\mathbb{T})\right)$ is a weak solution of NLS (1.1) in the extended sense if (i) $\left.u\right|_{t=0}=u_{0}$, (ii) the nonlinearity $\mathcal{N}(u)$ exists in the sense of Definition 1.2, and (iii) $u$ satisfies (1.1) in the sense of distributions on $\mathbb{T} \times(0, T)$, where the nonlinearity $\mathcal{N}(u)=u|u|^{2}$ is interpreted as above.

In the following, we construct weak solutions of (1.1) with $u_{0} \in L^{2}(\mathbb{T})$, by directly establishing an energy estimate in $C\left([0, T] ; L^{2}\right)$ without using any auxiliary function space. Our first result is the following. 
Theorem 1.4 (Existence). Let $s \geq 0$. Then, for $u_{0} \in H^{s}(\mathbb{T})$, there exists a weak solution $u \in C\left([0, T] ; H^{s}(\mathbb{T})\right)$ of $N L S$ (1.1) with initial condition $u_{0}$ in the sense of Definition 1.3, where the time $T$ of existence depends only on $\left\|u_{0}\right\|_{H^{s}}$. Moreover, the solution map is Lipschitz continuous.

Remark 1.5. In view of the embedding $H^{s}(\mathbb{T}) \subset L^{3}(\mathbb{T})$ for $s \geq \frac{1}{6}$, it follows that when $s \geq \frac{1}{6}$, the solution $u$ in Theorem 1.4 indeed satisfies NLS (1.1) in the usual distributional sense as a space-time distribution. Moreover, for each fixed $t \in(0, T)$, it satisfies the equation (1.1) as a spatial distribution on $\mathbb{T}$.

In Theorem 1.4, the uniqueness holds only as a limit of classical solutions. This in particular implies that the solutions in Theorem 1.4 coincide with the solutions constructed in Bourgain's $L^{2}$ well-posedness result [3]. Hence, they lie in the class

$$
C\left([0, T] ; L^{2}(\mathbb{T})\right) \cap X_{T}^{0, \frac{3}{8}} \subset C\left([0, T] ; L^{2}(\mathbb{T})\right) \cap L_{x, T}^{4},
$$

where $X_{T}^{0, \frac{3}{8}}$ denotes the local-in-time version of $X^{0, \frac{3}{8}}$ and $L_{x, T}^{4}=L^{4}(\mathbb{T} \times[0, T])$.

Now, let us turn to the uniqueness statement of solutions of (1.1). Recall the following definition from Kato 19. We say that a Cauchy problem is unconditionally well-posed in $H^{s}$ if for every initial condition $u_{0} \in H^{s}$, there exist $T>0$ and a unique solution $u \in C\left([0, T] ; H^{s}\right)$ such that $u(0)=u_{0}$. Also, see [13. We refer to such uniqueness in $C\left([0, T] ; H^{s}\right)$ without intersecting with any auxiliary function space as unconditional uniqueness. Unconditional uniqueness is a concept of uniqueness which does not depend on how solutions are constructed.

As mentioned above, the $L^{2}$ well-posedness result in [3] assumes that solutions are a priori in $X^{0, \frac{3}{8}}$ (locally in time) due to the use of the periodic $L^{4}$ Strichartz estimate (1.3). Thus, the uniqueness in [3] holds only in the class (1.5). Namely, the uniqueness of solutions in [3] holds conditionally, since uniqueness may not hold without the restriction of the auxiliary function space $X_{T}^{0, \frac{3}{8}}$.

The proof of Theorem 1.4 only uses a direct energy estimate in $C\left([0, T] ; L^{2}\right)$. For a general solution $u \in C\left([0, T] ; L^{2}\right)$, we need to perform the argument through an approximating smooth solution $u_{n}$. However, if $u \in C\left([0, T] ; H^{s}\right)$ for some $s \geq \frac{1}{6}$, we do not need such an approximating sequence of smooth solutions and directly work on $u$. This yields the following uniqueness of solutions to (1.1) in $H^{s}(\mathbb{T}), s \geq \frac{1}{6}$.

Theorem 1.6 (Unconditional uniqueness). Let $s \geq \frac{1}{6}$. Then, for $u_{0} \in H^{s}(\mathbb{T})$, the solution $u$ with initial condition $u_{0}$ constructed in Theorem 1.4 is unique in $C\left([0, T] ; H^{s}(\mathbb{T})\right)$.

Theorem 1.6 with the $L^{2}$-conservation law for (1.1) yields the following corollary.

Corollary 1.7. Let $s \geq \frac{1}{6}$. NLS (1.1) is unconditionally globally well-posed in $H^{s}(\mathbb{T})$.

Note that Theorem 1.6] is an improvement of Bourgain's result [3] in the aspect of uniqueness, at least for $s \geq \frac{1}{6}$. We also point out that, for $s<0$, there is the non-uniqueness result in $C_{t} H^{s}(\mathbb{T})$ by Christ [7] (for solutions in extended sense as in Definition 1.3.) See [7] for details.

Many of the unconditional uniqueness results use some auxiliary function spaces (such as Strichartz spaces and $X^{s, b}$ spaces), which are designed to be large enough to contain $C\left([0, T] ; H^{s}\right)$ such that desired nonlinear estimates hold. See, for instance, Zhou [23]. However, we simply use the $C\left([0, T] ; H^{s}\right)$-norm in the proof of Theorem 1.6 . 
For $s>\frac{1}{2}$, an a priori estimate in $C\left([0, T] ; H^{s}\right)$ easily follows from Sobolev embedding theorem. The challenge is to go below this regularity. We achieve this goal by implementing an infinite iteration scheme for the Poincaré-Dulac normal form reductions. See Subsection 1.2 for a discussion on the Poincaré-Dulac normal form reduction. This method provides a new method to prove well-posedness of PDEs.

In [8], Christ used the power series argument to construct solutions to the (renormalized) cubic NLS in $C\left([0, T] ; \mathcal{F} L^{s, p}\right), s \geq 0, p \in[1, \infty)$, where the Fourier-Lebesgue space $\mathcal{F} L^{s, p}$ is defined by the norm $\|f\|_{\mathcal{F} L^{s, p}}=\left\|\langle n\rangle^{s} \widehat{f}(n)\right\|_{\ell_{n}^{p}}$. This argument involves a power series expansion of solutions in terms of initial data and uses only the $C\left([0, T] ; \mathcal{F} L^{s, p}\right)$-norm. However, this construction does not provide uniqueness 1 When $p=2$, a slight modification of his argument can be applied to the original cubic NLS (1.1) for construction of solutions in $C\left([0, T] ; H^{s}\right), s \geq 0$ (without any auxiliary norms.) It may be of interest to compare and possibly combine two infinite iteration arguments in [8] and this paper.

We prove Theorems 1.4 and 1.6 by establishing a priori estimates, where we only use the $C_{t} H_{x}^{s}$-norm of solutions. In the next subsection, we briefly describe the idea of PoincaréDulac normal form reductions.

Before doing so, let us introduce an equivalent formulation to (1.1). Let $S(t)=e^{-i t \partial_{x}^{2}}$ denote the semigroup to the linear Schrödinger equation: $i u_{t}-u_{x x}=0$. We apply a change of coordinates: $v(t)=S(-t) u(t)=e^{i t \partial_{x}^{2}} u(t)$, i.e. $\widehat{v}(n, t)=e^{-i n^{2} t} \widehat{u}(n, t)$. For simplicity of notation, we use $v_{n}=v_{n}(t)$ to denote $\widehat{v}(n, t)$. Then, $v$ satisfies the following equation:

$$
\begin{aligned}
\partial_{t} v_{n}(t) & =i \mathfrak{N}(v, v, v)(n, t) \\
& =i \sum_{\substack{n=n_{1}-n_{2}+n_{3}\\
}} e^{-i \Phi(\bar{n}) t} v_{n_{1}} \bar{v}_{n_{2}} v_{n_{3}} \\
& =i \sum_{\substack{n=n_{1}-n_{2}+n_{3} \\
n_{2} \neq n_{1}, n_{3}}} e^{-i \Phi(\bar{n}) t} v_{n_{1}} \bar{v}_{n_{2}} v_{n_{3}}+i \sum_{\substack{n=n_{1}-n_{2}+n_{3} \\
n_{2}=n_{1} \text { or } n_{3}}} e^{-i \Phi(\bar{n}) t} v_{n_{1}} \bar{v}_{n_{2}} v_{n_{3}} \\
& =: i \mathfrak{N}_{1}(v, v, v)(n, t)+i \mathfrak{R}_{1}(v, v, v)(n, t) .
\end{aligned}
$$

Note that $v(0)=u_{0} \in H^{s}(\mathbb{T})$. The phase function $\Phi(\bar{n})$ is defined by

$$
\begin{aligned}
\Phi(\bar{n}): & =\Phi\left(n, n_{1}, n_{2}, n_{3}\right)=n^{2}-n_{1}^{2}+n_{2}^{2}-n_{3}^{2} \\
& =2\left(n_{2}-n_{1}\right)\left(n_{2}-n_{3}\right)=2\left(n-n_{1}\right)\left(n-n_{3}\right),
\end{aligned}
$$

where the last two equalities hold under $n=n_{1}-n_{2}+n_{3}$. From (1.8), it follows that $\mathfrak{N}_{1}$ corresponds to the non-resonant part (i.e. $\Phi(\bar{n}) \neq 0$ ) of the nonlinearity. Throughout this paper, we introduce several multilinear expressions.2 We often suppress its dependence on $t$ and its multiple arguments $v$. For example, we write $\left(\mathfrak{N}_{1}\right)_{n}$ or $\mathfrak{N}_{1}(v)_{n}$ for $\mathfrak{N}_{1}(v, v, v)(n, t)$.

As is well known, working in terms of $v$ has certain advantages. In [3], Bourgain made an effective use of this coordinate (called interaction representation in Quantum Mechanics [15]) by introducing the $X^{s, b}$ spaces. From the definition (1.2), we have $\|u\|_{X^{s, b}}=\|v\|_{H_{t}^{b} H_{x}^{s}}$, i.e. a function $u$ is in $X^{s, b}$ if and only if its interaction representation $v(t)=S(-t) u(t)$ is in the classical Sobolev space $H_{t}^{b} H_{x}^{s}$.

\footnotetext{
${ }^{1}$ Grünrock-Herr 16] proved (conditional) local well-posedness in $\mathcal{F} L^{s, p}, s \geq 0, p \in(2, \infty)$ via the fixed point argument. The uniqueness in [16] holds only in $C\left([0, T] ; \mathcal{F} L^{s, p}\right)$ intersected with the $X^{s, b}$ space adapted to $\mathcal{F} L^{s, p}$.

${ }^{2} \mathrm{By}$ a multilinear operator, we mean an operator such that it is linear or conjugate linear with respect to each argument.
} 
1.2. Poincaré-Dulac normal form reduction: formal argument. First, let us describe the classical Poincaré-Dulac Theorem. Consider a formal vector-valued power series $A x+F(x):=A x+\sum_{j=a}^{\infty} f_{j}(x)$, with some $a \geq 2$, in $n$ variables $x=\left(x_{1}, x_{2}, \cdots, x_{n}\right)$, where $f_{j}(x)$ denotes nonlinear terms of degree $j$ in $x$. Assume that the eigenvalues of $A$ are distinct. Then, Poincaré-Dulac Theorem [1] states the following. Given a differential equation

$$
\partial_{t} x=A x+F(x)=A x+\sum_{j=a}^{\infty} f_{j}(x)
$$

we can introduce a sequence of formal changes of variables

$$
\begin{aligned}
& z_{1}=x+y_{1}, \\
& z_{2}=z_{1}+y_{2}=x+y_{1}+y_{2}, \\
& \quad \vdots \\
& z=z_{\infty}=x+\sum_{j=1}^{\infty} y_{j},
\end{aligned}
$$

to reduce the system to the canonical form:

$$
\partial_{t} z=A z+G(z)=A z+\sum_{j=a}^{\infty} g_{j}(z)
$$

where $g_{j}(z)$ in the series $G(z)=\sum_{j=a}^{\infty} g_{j}(z)$ denotes resonant monomials of degree $j$ in $z 3$ Moreover, after the $k$ th step, we have

$$
\partial_{t} z_{k}=A z_{k}+G_{k}\left(z_{k}\right)
$$

where monomials of degree up to $k(a-1)+a-2$ in $G_{k}\left(z_{k}\right)$ are all resonant.

With $\widetilde{x}(t)=e^{-t A} x(t), \widetilde{y}_{j}(t)=e^{-t A} y_{j}(t)$, and so on, namely "working in terms of the interaction representations," we can rewrite the original equation (1.9) as

$$
\partial_{t} \widetilde{x}=e^{-t A} F\left(e^{t A} \widetilde{x}\right),
$$

and the resulting canonical equations (1.12) and (1.11) as

$$
\begin{cases}\partial_{t} \widetilde{z}_{k}=e^{-t A} G_{k}\left(e^{t A} \widetilde{z}_{k}\right), & \text { after the } k \text { th step, } \\ \partial_{t} \widetilde{z}=e^{-t A} G\left(e^{t A} \widetilde{z}\right), & k=\infty\end{cases}
$$

Note that the right hand sides of (1.14) consist of only resonant monomials when $k=\infty$ (and up to degree $k(a-1)+a-2$ after the $k$ th step.) After integrating (1.14) in time, we obtain

$$
\begin{cases}\widetilde{z}_{k}(t)=\widetilde{z}_{k}(0)+\int_{0}^{t} e^{-t^{\prime} A} G_{k}\left(e^{t^{\prime} A} \widetilde{z}_{k}\left(t^{\prime}\right)\right) d t^{\prime}, & \text { after the } k \text { th step } \\ \widetilde{z}(t)=\widetilde{z}(0)+\int_{0}^{t} e^{-t^{\prime} A} G\left(e^{t^{\prime} A} \widetilde{z}\left(t^{\prime}\right)\right) d t^{\prime}, & k=\infty\end{cases}
$$

With (1.10), we formally have

(1) After the $k$ th step:

$$
\widetilde{x}(t)=\widetilde{x}(0)-\sum_{j=1}^{k}\left[\widetilde{y}_{j}(t)-\widetilde{y}_{j}(0)\right]+\int_{0}^{t} e^{-t^{\prime} A} G_{k}\left(e^{\left.t^{\prime} A_{z_{k}}\left(t^{\prime}\right)\right) d t^{\prime} .}\right.
$$

\footnotetext{
${ }^{3}$ In this formal discussion, we intentionally remain vague about the definition of resonant monomials. See Arnold [1] for the precise definition.
} 
Recall that monomials of degree up to $k(a-1)+a-2$ in $G_{k}\left(z_{k}\right)$ are all resonant.

(2) With $k=\infty$ :

$$
\widetilde{x}(t)=\widetilde{x}(0)-\sum_{j=1}^{\infty}\left[\widetilde{y}_{j}(t)-\widetilde{y}_{j}(0)\right]+\int_{0}^{t} e^{-t^{\prime} A} G\left(e^{t^{\prime} A} \widetilde{z}\left(t^{\prime}\right)\right) d t^{\prime} .
$$

The point of the classical Poincaré-Dulac normal form is to renormalize the flow so that it is expressed in terms of resonant terms as in (1.12), (1.14), and (1.15). However, for our purpose, the formulations (1.16) and (1.17) turn out to be more useful.

In the following, we take the infinite dimensional system (1.6), and formally apply the Poincaré-Dulac normal form reductions to it. In order to prove Theorems 1.4 and 1.6, we present the revised application of the Poincaré-Dulac normal form reductions with estimates in Sections 2 and 3 ,

The term $\mathfrak{R}_{1}(v)$ in (1.6) consists of only resonant monomials, and thus we leave it as it is. Now, apply differentiation by parts, i.e. integration by parts without an integration symbol - this terminology was introduced in Babin-Ilyin-Titi [2] - on the non-resonant part $\mathfrak{N}_{1}(v)$ :

$$
\begin{aligned}
\left(\mathfrak{N}_{1}(v)\right)_{n}= & \partial_{t}\left[i \sum_{\substack{n=n_{1}-n_{2}+n_{3} \\
n_{2} \neq n_{1}, n_{3}}} \frac{e^{-i \Phi(\bar{n}) t}}{\Phi(\bar{n})} v_{n_{1}} \bar{v}_{n_{2}} v_{n_{3}}\right] \\
& -i \sum_{\substack{n=n_{1}-n_{2}+n_{3} \\
n_{2} \neq n_{1}, n_{3}}} \frac{e^{-i \Phi(\bar{n}) t}}{\Phi(\bar{n})} \partial_{t}\left(v_{n_{1}} \bar{v}_{n_{2}} v_{n_{3}}\right) \\
= & : \partial_{t}\left(\mathfrak{N}_{21}\right)_{n}+\left(\mathfrak{N}_{22}\right)_{n} .
\end{aligned}
$$

For simplicity of presentation, let us drop the complex number $i$ and simply use 1 for \pm 1 and $\pm i$ appearing in the following formal computation. Moreover, assume that the time derivative falls on the first factor $v_{n_{1}}$ of $v_{n_{1}} \bar{v}_{n_{2}} v_{n_{3}}$ in the second term $\mathfrak{N}_{22}$ in (1.18), counting the multiplicity. Then, from (1.6), we have

$$
\begin{aligned}
\left(\mathfrak{N}_{22}\right)_{n} & =3 \sum_{\substack{n=n_{1}-n_{2}+n_{3} \\
n_{2} \neq n_{1}, n_{3}}} \frac{e^{-i \Phi(\bar{n}) t}}{\Phi(\bar{n})}(\mathfrak{N})_{n_{1}} \bar{v}_{n_{2}} v_{n_{3}} \\
& =3 \sum_{\substack{n=n_{1}-n_{2}+n_{3} \\
n_{2} \neq n_{1}, n_{3}}} \sum_{n_{1}=m_{1}-m_{2}+m_{3}} \frac{e^{-i(\Phi(\bar{n})+\Phi(\bar{m})) t}}{\Phi(\bar{n})} v_{m_{1}} \bar{v}_{m_{2}} v_{m_{3}} \bar{v}_{n_{2}} v_{n_{3}} .
\end{aligned}
$$

As before, the phase function $\Phi(\bar{m})$ is defined by

$$
\begin{aligned}
\Phi(\bar{m}): & =\Phi\left(n_{1}, m_{1}, m_{2}, m_{3}\right)=n_{1}^{2}-m_{1}^{2}+m_{2}^{2}-m_{3}^{2} \\
& =2\left(m_{2}-m_{1}\right)\left(m_{2}-m_{3}\right)=2\left(n_{1}-m_{1}\right)\left(n_{1}-m_{3}\right),
\end{aligned}
$$

where the last two equalities hold under $n_{1}=m_{1}-m_{2}+m_{3}$.

In particular, $\mathfrak{N}_{22}$ consists of quintic monomials. Then, from (1.6), (1.18), and (1.19), we have

$$
v(t)=v(0)+\mathfrak{N}_{21}(t)-\mathfrak{N}_{21}(0)+\int_{0}^{t} \mathfrak{R}_{1}\left(t^{\prime}\right)+\mathfrak{N}_{22}\left(t^{\prime}\right) d t^{\prime} .
$$

This corresponds to (1.16) with $k=1$ (and $a=3$.) Indeed, all of the cubic monomials in the integrand of (1.21) are in $\mathfrak{R}_{1}$, and they are all resonant, verifying the condition 
$3=k+a-1$ with $k=1$ and $a=3$. Also, $\mathfrak{N}_{21}$ corresponds to the first correction term $\widetilde{y_{1}}$ and its degree is 3 .

In the second step, we can divide $\mathfrak{N}_{22}$ into its resonant part $\mathfrak{R}_{1}$ and non-resonant part $\mathfrak{N}_{2}$, according to $\Phi(\bar{n})+\Phi(\bar{m})=0$ or $\neq 0$. Then, we apply differentiation by parts on the non-resonant part $\mathfrak{N}_{2}$. This yields

$$
\mathfrak{N}_{2}=\partial_{t} \mathfrak{N}_{31}+\mathfrak{N}_{32}
$$

where $\mathfrak{N}_{31}$ consists of quintic monomials and $\mathfrak{N}_{32}$ consists of septic monomials. Moreover, the constant appearing in front of the summation is $3 \cdot 5$. (We assume that the time derivative falls on the first of the five factors, and thus we need to count the multiplicity.) See (1.19). Then, from (1.6), (1.18), and (1.22), we have

$$
v(t)=v(0)+\sum_{j=1}^{2}\left[\mathfrak{N}_{(j+1) 1}(t)-\mathfrak{N}_{(j+1) 1}(0)\right]+\int_{0}^{t} \mathfrak{R}_{1}\left(t^{\prime}\right)+\mathfrak{R}_{2}\left(t^{\prime}\right)+\mathfrak{N}_{32}\left(t^{\prime}\right) d t^{\prime},
$$

corresponding to (1.16) with $k=2$ (and $a=3$.) Since $\mathfrak{N}_{32}$ consists of septic terms, all the terms up to degree 5 in the integrand in (1.23) are in $\mathfrak{R}_{1}$ or $\mathfrak{R}_{2}$, and hence they are resonant.

In this way, we can repeat this formal procedure indefinitely. However, for our purpose, we need to estimate each term in $C_{t} H^{s}$, and there are three potential difficulties.

(1) We need to estimate higher and higher order monomials. This corresponds to establishing multilinear estimates with higher and higher degrees of nonlinearities.

(2) At the $k$ th step, the number of factors on which the time derivative falls is $2 k+1$. Thus, the constants grow like $3 \cdot 5 \cdot 7 \cdots \cdots(2 k+1)$.

(3) Our multilinear estimates need to provide small constants on the terms without time integration, i.e. on the boundary terms, such as $\mathfrak{N}_{21}(t)-\mathfrak{N}_{21}(0)$ in (1.21) and $\sum_{j=1}^{2}\left[\mathfrak{N}_{(j+1) 1}(t)-\mathfrak{N}_{(j+1) 1}(0)\right]$ in $(1.23)$. (We can introduce small constants for the terms inside time integration by making the time interval of integration sufficiently small, depending on $\left\|u_{0}\right\|_{L^{2}}$.)

In the following two sections, we revise this formal iteration of Poincaré-Dulac normal form reductions to treat these three issues. In particular, when we apply differentiation by parts on the non-resonant part $\mathfrak{N}_{k}$ consisting of monomials of degree $2 k+1$, we first divide it into two parts: a part on which we can directly establish $(2 k+1)$-linear estimate (without differentiation by parts) and a part on which we can not establish any $(2 k+1)$-linear estimate. Then, we apply differentiation by parts on the second part. The issues (2) and (3) can be treated by introducing different levels of thresholds for separating resonant and non-resonant parts at each iteration step. See (2.5), (3.6), (3.17), and (3.33). Lastly, we point out that the crucial tool for establishing multilinear estimates is the divisor counting argument (as in the proof of the periodic $L^{4}$ - and $L^{6}$-Strichartz estimates by Bourgain [3].) See (2.6).

A precursor to this argument appears in the work by Babin-Ilyin-Titi 2 for $\mathrm{KdV}$ on $\mathbb{T}$, followed by the authors [20] for $\mathrm{mKdV}$ on $\mathbb{T} 4$ Note that two iterations were sufficient in [2, 20] (in [20], the second differentiation by parts is performed in a slightly different manner in the endpoint case) whereas, for cubic NLS, we need to iterate the argument infinitely many times. This is perhaps due to weaker dispersion of the Schrödinger equation as compared to that of the Airy equation (= linear part of KdV and mKdV.) Also, Shatah [21]

\footnotetext{
${ }^{4}$ This kind of integration by parts was previously used in Takaoka-Tsutsumi 22 .
} 
and, more recently, Germain-Masmoudi-Shatah [14] use ideas from Poincaré-Dulac normal form reduction 5 (to send a quadratic term into a cubic one by one iteration.) However, their goal is global-in-time behavior of small solutions, and is different from ours (local-in-time with large data.)

Note that the Poincaré-Dulac normal form can be (formally) applied to non-Hamiltonian equations, whereas the Birkhoff normal form is for Hamiltonian equations. See Bourgain [4, 5] and Colliander-Kwon-Oh [1] for inductive argument on the application of the Birkhoff normal form. We point out that the argument in [4, 5, 11] is for large times with finite numbers of iterations, whereas our argument is local-in-time with an infinite number of iterations.

This paper is organized as follows. In Section 2, we present the first step of (a revised version of) Poincaré-Dulac normal form reduction along with relevant estimates. In Section 3. we introduce some notations and implement an infinite iteration scheme of (a revised version of) Poincaré-Dulac normal form reductions, establishing estimates on the terms appearing at each step. In Section 4, we first express a smooth solution as a sum of infinite series (see (4.2) ), and make sense of such a representation by the estimates in Sections 2 and 3. Then, we construct a weak solution in $C\left([0, T] ; L^{2}\right)$ with initial condition in $L^{2}$. In Section 5, we work on $H^{s}$ for $s \geq \frac{1}{6}$ and justify the formal argument in Sections 2 and 3 , This proves unconditional uniqueness in $C\left([0, T] ; H^{s}\right)$ for $s \geq \frac{1}{6}$.

\section{Poincaré-Dulac normal form Reduction, Part 1: Basic Setup}

In this section, we discuss the first step of Poincaré-Dulac normal form reduction. In the following, we take $s=0$ for simplicity, and estimate each multilinear expression appearing in the discussion by the $L_{x}^{2}$-norm, independent of time. Namely, we establish direct $C_{t} L_{x}^{2}$ estimates. Then, we implement an infinite iteration scheme in the next section. As in Section 1, we often drop the complex number $i$ and simply use 1 for \pm 1 and $\pm i$ in the following 6 Lastly, in Sections 2 and 3, we perform all the formal computations, assuming that $u$ (and hence $v$ ) is a smooth solution. In Section 5, we justify our formal computations when $u \in C_{t} H^{s}, s \geq \frac{1}{6}$.

First, we write the nonlinearity $u|u|^{2}$ in (1.1) as

$$
\begin{aligned}
u|u|^{2}= & \left(u|u|^{2}-2 u f_{\mathbb{T}}|u|^{2} d x\right)+2 u f_{\mathbb{T}}|u|^{2} d x \\
= & \sum_{n_{2} \neq n_{1}, n_{3}} \widehat{u}\left(n_{1}\right) \overline{u\left(n_{2}\right)} \widehat{u}\left(n_{3}\right) e^{i\left(n_{1}-n_{2}+n_{3}\right) x}-\sum_{n} \widehat{u}(n)|\widehat{u}(n)|^{2} e^{i n x} \\
& +2\left(f_{\mathbb{T}}|u|^{2} d x\right) \sum_{n} \widehat{u}(n) e^{i n x},
\end{aligned}
$$

\footnotetext{
${ }^{5}$ In [14], they introduced time resonances, space resonances, and space-time resonances. Resonances in this paper correspond to their time resonances.

${ }^{6}$ When we apply differentiation by parts, we keep the minus sign on the second term for emphasis. For example, see (2.10).
} 
where $f_{\mathbb{T}}|u|^{2} d x:=\frac{1}{2 \pi} \int_{\mathbb{T}}|u|^{2} d x$. Then, (1.6) can be written as

$$
\begin{aligned}
\partial_{t} v_{n} & =i \sum_{\substack{n=n_{1}-n_{2}+n_{3} \\
n_{2} \neq n_{1}, n_{3}}} e^{-i \Phi(\bar{n}) t} v_{n_{1}} \bar{v}_{n_{2}} v_{n_{3}}-i\left|v_{n}\right|^{2} v_{n}+2 i\left(f_{\mathbb{T}}|v|^{2} d x\right) v_{n} \\
& =: i \mathcal{N}_{1}(v)(n)-i \mathcal{R}_{1}(v)(n)+i \mathcal{R}_{2}(v)(n),
\end{aligned}
$$

where the phase function $\Phi(\bar{n})$ is as in (1.8). From (1.8), it follows that $\mathcal{N}_{1}$ corresponds to the non-resonant part (i.e. $\Phi(\bar{n}) \neq 0$ ) of the nonlinearity and $\mathcal{R}_{1}$ and $\mathcal{R}_{2}$ correspond to the resonant part.

Lemma 2.1. Let $\mathcal{R}_{1}$ and $\mathcal{R}_{2}$ be as in (2.1). Then, we have

$$
\begin{aligned}
\left\|\mathcal{R}_{j}(v)\right\|_{L^{2}} & \lesssim\|v\|_{L^{2}}^{3}, \\
\left\|\mathcal{R}_{j}(v)-\mathcal{R}_{j}(w)\right\|_{L^{2}} & \lesssim\left(\|v\|_{L^{2}}^{2}+\|w\|_{L^{2}}^{2}\right)\|v-w\|_{L^{2}}
\end{aligned}
$$

for $j=1,2$.

Proof. For $\mathcal{R}_{1}$, this is clear from $\ell_{n}^{2} \subset \ell_{n}^{6}$. For $\mathcal{R}_{2}$, the result follows from Cauchy-Schwarz inequality, once we note

$$
\begin{aligned}
& 2\left(\int_{\mathbb{T}}|v|^{2} d x\right) v_{n}-2\left(\int_{\mathbb{T}}|w|^{2} d x\right) w_{n} \\
& \quad=2\left(\int_{\mathbb{T}} v(\bar{v}-\bar{w}) d x+\int_{\mathbb{T}}(v-w) \bar{w} d x\right) v_{n}+2\left(\int_{\mathbb{T}}|v|^{2} d x\right)\left(v_{n}-w_{n}\right) .
\end{aligned}
$$

Next, we consider the non-resonant part $\mathcal{N}_{1}$. Let $N>0$ be a large parameter. (As we see later, $N=N\left(\left\|u_{0}\right\|_{L^{2}}\right)$.) First, we write

$$
\mathcal{N}_{1}=\mathcal{N}_{11}+\mathcal{N}_{12}
$$

where $\mathcal{N}_{11}$ is the restriction of $\mathcal{N}_{1}$ onto $A_{N}$, where $A_{N}=\bigcup_{n} A_{N}(n)$ with

$$
\begin{aligned}
A_{N}(n):=\left\{\left(n, n_{1}, n_{2}, n_{3}\right)\right. & ;=n_{1}-n_{2}+n_{3}, n_{1}, n_{3} \neq n, \\
& \left.|\Phi(\bar{n})|=\left|2\left(n-n_{1}\right)\left(n-n_{3}\right)\right| \leq N\right\}
\end{aligned}
$$

and $\mathcal{N}_{12}:=\mathcal{N}_{1}-\mathcal{N}_{11}$

Recall the following number theoretic fact [17]. Given an integer $m$, let $d(m)$ denote the number of divisors of $m$. Then, we have

$$
d(m) \lesssim e^{c \frac{\log m}{\log \log m}}\left(=o\left(m^{\varepsilon}\right) \text { for any } \varepsilon>0 .\right)
$$

With (2.6), we estimate $\mathcal{N}_{11}$ as follows.

Lemma 2.2. Let $\mathcal{N}_{11}$ be as above. Then, we have

$$
\begin{aligned}
\left\|\mathcal{N}_{11}(v)\right\|_{L^{2}} & \lesssim N^{\frac{1}{2}+}\|v\|_{L^{2}}^{3}, \\
\left\|\mathcal{N}_{11}(v)-\mathcal{N}_{11}(w)\right\|_{L^{2}} & \lesssim N^{\frac{1}{2}+}\left(\|v\|_{L^{2}}^{2}+\|w\|_{L^{2}}^{2}\right)\|v-w\|_{L^{2}} .
\end{aligned}
$$

Proof. We only prove (2.7) since (2.8) follows in a similar manner. Fix $n, \mu \in \mathbb{Z}$ with $|\mu| \leq N$. Then, from (2.6), there are at most $o\left(N^{0+}\right)$ many choices for $n_{1}$ and $n_{3}$ (and hence for $n_{2}$ from $\left.n=n_{1}-n_{2}+n_{3}\right)$ satisfying

$$
\mu=2\left(n-n_{1}\right)\left(n-n_{3}\right) .
$$


Then, by Cauchy-Schwarz inequality, we have

$$
\begin{aligned}
\left\|\mathcal{N}_{11}\right\|_{L^{2}} & =\left(\sum_{n}\left|\sum_{\substack {|\mu| \leq N \\
\begin{subarray}{c}{n=n_{1}-n_{2}+n_{3} \\
n_{2} \neq n_{1}, n_{3} \\
\mu=\Phi(\bar{n}){ | \mu | \leq N \\
\begin{subarray} { c } { n = n _ { 1 } - n _ { 2 } + n _ { 3 } \\
n _ { 2 } \neq n _ { 1 } , n _ { 3 } \\
\mu = \Phi ( \overline { n } ) } }\end{subarray}} v_{n_{1}} \bar{v}_{n_{2}} v_{n_{3}}\right|^{2}\right)^{\frac{1}{2}} \\
& \leq\left\{\sum_{n}\left(\sum_{|\mu| \leq N} N^{0+}\right)\left(\sum_{\substack{n=n_{1}-n_{2}+n_{3} \\
n_{1}, n_{3}}}\left|v_{n_{1}}\right|^{2}\left|v_{n_{1}+n_{3}-n}\right|^{2}\left|v_{n_{3}}\right|^{2}\right)\right\}^{\frac{1}{2}} \\
& \lesssim N^{\frac{1}{2}+}\|v\|_{L^{2}}^{3} .
\end{aligned}
$$

Now, we apply (the first step of) Poincaré-Dulac normal form reduction to the remaining non-resonant part $\mathcal{N}_{12}$. Namely, we differentiate $\mathcal{N}_{12}$ by parts (i.e. apply the product rule on differentiation in a reversed order) and write

$$
\begin{aligned}
\mathcal{N}_{12}(v)_{n}= & \sum_{A_{N}(n)^{c}} \partial_{t}\left(\frac{e^{-i \Phi(\bar{n}) t}}{-i \Phi(\bar{n})}\right) v_{n_{1}} \bar{v}_{n_{2}} v_{n_{3}} \\
= & i \sum_{A_{N}(n)^{c}} \partial_{t}\left[\frac{e^{-i \Phi(\bar{n}) t}}{2\left(n-n_{1}\right)\left(n-n_{3}\right)} v_{n_{1}} \bar{v}_{n_{2}} v_{n_{3}}\right] \\
& \quad-i \sum_{A_{N}(n)^{c}} \frac{e^{-i \Phi(\bar{n}) t}}{2\left(n-n_{1}\right)\left(n-n_{3}\right)} \partial_{t}\left(v_{n_{1}} \bar{v}_{n_{2}} v_{n_{3}}\right) \\
= & i \partial_{t}\left[\sum_{A_{N}(n)^{c}} \frac{e^{-i \Phi(\bar{n}) t}}{2\left(n-n_{1}\right)\left(n-n_{3}\right)} v_{n_{1}} \bar{v}_{n_{2}} v_{n_{3}}\right] \\
& \quad-i \sum_{A_{N}(n)^{c}} \frac{e^{-i \Phi(\bar{n}) t}}{2\left(n-n_{1}\right)\left(n-n_{3}\right)} \partial_{t}\left(v_{n_{1}} \bar{v}_{n_{2}} v_{n_{3}}\right) \\
= & : \partial_{t}\left(\mathcal{N}_{21}\right)_{n}+\left(\mathcal{N}_{22}\right)_{n} .
\end{aligned}
$$

Note that we formally exchanged the order of the sum and the time differentiation in the first term at the third equality. See Section 5 for more on this issue.

In the following, we assume that the frequencies $\left(n, n_{1}, n_{2}, n_{3}\right)$ are on $A_{N}^{c}$ defined in (2.5), and we may not state it explicitly.

Lemma 2.3. Let $\mathcal{N}_{21}$ be as in (2.10). Then, we have

$$
\begin{aligned}
\left\|\mathcal{N}_{21}(v)\right\|_{L^{2}} & \lesssim N^{-\frac{1}{2}+}\|v\|_{L^{2}}^{3}, \\
\left\|\mathcal{N}_{21}(v)-\mathcal{N}_{21}(w)\right\|_{L^{2}} & \lesssim N^{-\frac{1}{2}+}\left(\|v\|_{L^{2}}^{2}+\|w\|_{L^{2}}^{2}\right)\|v-w\|_{L^{2}}
\end{aligned}
$$

Proof. We only prove (2.11) since (2.12) follows in a similar manner. On $A_{N}^{c}$, we have $|\mu|>N$ where $\mu$ is as in (2.9). As before, for fixed $n, \mu \in \mathbb{Z}$, there are at most $o\left(|\mu|^{0+}\right)$ many choices for $n_{1}$ and $n_{3}$. Then, by Cauchy-Schwarz inequality, we have

$$
\begin{aligned}
\left\|\mathcal{N}_{21}\right\|_{L^{2}} & \lesssim\left\{\sum_{n}\left(\sum_{|\mu|>N} \frac{1}{|\mu|^{2}}|\mu|^{0+}\right)\left(\sum_{n_{1}, n_{3}}\left|v_{n_{1}}\right|^{2}\left|\bar{v}_{n_{2}}\right|^{2}\left|v_{n_{3}}\right|^{2}\right)\right\}^{\frac{1}{2}} \\
& \lesssim N^{-\frac{1}{2}+}\|v\|_{L^{2}}^{3} .
\end{aligned}
$$


By symmetry between $n_{1}$ and $n_{3}$, we can write the remaining term $\mathcal{N}_{22}$ as

$$
\begin{aligned}
\left(\mathcal{N}_{22}\right)_{n}= & -2 i \sum_{\substack{n=n_{1}-n_{2}+n_{3} \\
n_{2} \neq n_{1}, n_{3}}} \frac{e^{-i \Phi(\bar{n}) t}}{2\left(n-n_{1}\right)\left(n-n_{3}\right)} \partial_{t} v_{n_{1}} \bar{v}_{n_{2}} v_{n_{3}} \\
& -i \sum_{\substack{n=n_{1}-n_{2}+n_{3} \\
n_{2} \neq n_{1}, n_{3}}} \frac{e^{-i \Phi(\bar{n}) t}}{2\left(n-n_{1}\right)\left(n-n_{3}\right)} v_{n_{1}} \partial_{t} \bar{v}_{n_{2}} v_{n_{3}} \\
= & :\left(\mathcal{N}_{221}\right)_{n}+\left(\mathcal{N}_{222}\right)_{n} .
\end{aligned}
$$

In the following, we only estimate the first term $\mathcal{N}_{221}$ since $\mathcal{N}_{222}$ can be estimated analogously. From (2.1), $\mathcal{N}_{221}$ can be divided into two terms:

$$
\begin{aligned}
\left(\mathcal{N}_{221}\right)_{n}= & 2 \sum_{\substack{n=n_{1}-n_{2}+n_{3} \\
n_{2} \neq n_{1}, n_{3}}} \sum_{\substack{n_{1}=m_{1}-m_{2}+m_{3} \\
m_{2} \neq m_{1}, m_{3}}} \frac{e^{-i(\Phi(\bar{n})+\Phi(\bar{m})) t}}{2\left(n-n_{1}\right)\left(n-n_{3}\right)} v_{m_{1}} \bar{v}_{m_{2}} v_{m_{3}} \bar{v}_{n_{2}} v_{n_{3}} \\
& -2 \sum_{\substack{n=n_{1}-n_{2}+n_{3} \\
n_{2} \neq n_{1}, n_{3}}} \frac{e^{-i \Phi(\bar{n}) t}}{2\left(n-n_{1}\right)\left(n-n_{3}\right)}\left(\mathcal{R}_{1}-\mathcal{R}_{2}\right)_{n_{1}} \bar{v}_{n_{2}} v_{n_{3}} \\
= & :\left(\mathcal{N}_{3}\right)_{n}+\left(\mathcal{N}_{4}\right)_{n},
\end{aligned}
$$

where the phase function $\Phi(\bar{m})$ is as in (1.20). The second term $\mathcal{N}_{4}$ can be easily estimated.

Lemma 2.4. Let $\mathcal{N}_{4}$ be as in (2.14). Then, we have

$$
\begin{aligned}
\left\|\mathcal{N}_{4}(v)\right\|_{L^{2}} & \lesssim N^{-\frac{1}{2}+}\|v\|_{L^{2}}^{5}, \\
\left\|\mathcal{N}_{4}(v)-\mathcal{N}_{4}(w)\right\|_{L^{2}} & \lesssim N^{-\frac{1}{2}+}\left(\|v\|_{L^{2}}^{4}+\|w\|_{L^{2}}^{4}\right)\|v-w\|_{L^{2}} .
\end{aligned}
$$

Proof. This lemma follows from Lemmata 2.3 and 2.1 .

Now, it remains to estimate $\mathcal{N}_{3}$. As in (2.4), we separate $\mathcal{N}_{3}$ into two parts, depending on the size of the phase $\Phi(\bar{n})+\Phi(\bar{m})$ (see (3.7) below), and estimate a part of $\mathcal{N}_{3}$, corresponding to "small" phase $\Phi(\bar{n})+\Phi(\bar{m})$, as in Lemma 2.2. See Lemma 3.7. Then, we apply (the second step of) Poincaré-Dulac normal form reduction to the remaining (non-resonant) part with "large" phase $\Phi(\bar{n})+\Phi(\bar{m})$. See (3.11). However, it turns out that in order to prove Theorems 1.4 and 1.6, we need to iterate this procedure infinitely many times. Hence, in the next section, we first set up a necessary machinery and perform such an infinite iteration to estimate $\mathcal{N}_{3}$.

\section{Poincaré-Dulac normal form Reduction, Part 2: infinite iteration}

3.1. Notations: index by trees. In this section, we apply Poincaré-Dulac normal form reductions infinitely many times to estimate

$$
\left(\mathcal{N}_{3}\right)_{n}=2 \sum_{\substack{n=n_{1}-n_{2}+n_{3} \\ n_{2} \neq n_{1}, n_{3}}} \sum_{\substack{n_{1}=m_{1}-m_{2}+m_{3} \\ m_{2} \neq m_{1}, m_{3}}} \frac{e^{-i(\Phi(\bar{n})+\Phi(\bar{m})) t}}{2\left(n-n_{1}\right)\left(n-n_{3}\right)} v_{m_{1}} \bar{v}_{m_{2}} v_{m_{3}} \bar{v}_{n_{2}} v_{n_{3}} .
$$

In order to do so, we need to set up some notations. In the following, the complex conjugate signs on $v_{n_{j}}$ do not play any significant role, and thus we drop the complex conjugate sign. We also assume that all the Fourier coefficients $v_{n_{j}}$ are non-negative. 
When we apply differentiation by parts, we obtain terms like $\mathcal{N}_{22}$ in (2.10), where the time derivative may fall on any of the factors $v_{n_{j}}$. In general, the structure of such terms can be very complicated, depending on where the time derivative falls. In the following, we introduce the notion of trees (in particular, of ordered trees in Definition 3.3) for indexing such terms and frequencies arising in the general steps of the Poincaré-Dulac normal form reductions. We point out that some of the definitions are similar, but that some are different from those in Christ [8].

Definition 3.1. Given a partially ordered set $\mathcal{T}$ with partial order $\leq$, we say that $b \in \mathcal{T}$ with $b \leq a$ and $b \neq a$ is a child of $a \in \mathcal{T}$, if $b \leq c \leq a$ implies either $c=a$ or $c=b$. If the latter condition holds, we also say that $a$ is the parent of $b$.

As in Christ [8], our trees in this paper refer to a particular subclass of usual trees with the following properties:

Definition 3.2. A tree $\mathcal{T}$ is a finite partially ordered set satisfying the following properties.

(i) Let $a_{1}, a_{2}, a_{3}, a_{4} \in \mathcal{T}$. If $a_{4} \leq a_{2} \leq a_{1}$ and $a_{4} \leq a_{3} \leq a_{1}$, then we have $a_{2} \leq a_{3}$ or $a_{3} \leq a_{2}$

(ii) A node $a \in \mathcal{T}$ is called terminal, if it has no child. A non-terminal node $a \in \mathcal{T}$ is a node with exactly three children denoted by $a_{1}, a_{2}$, and $a_{3}$,

(iii) There exists a maximal element $r \in \mathcal{T}$ (called the root node) such that $a \leq r$ for all $a \in \mathcal{T}$. We assume that the root node is non-terminal,

(iv) $\mathcal{T}$ consists of the disjoint union of $\mathcal{T}^{0}$ and $\mathcal{T}^{\infty}$, where $\mathcal{T}^{0}$ and $\mathcal{T}^{\infty}$ denote the collections of non-terminal nodes and terminal nodes, respectively.

Note that the number $|\mathcal{T}|$ of nodes in a tree $\mathcal{T}$ is $3 j+1$ for some $j \in \mathbb{N}$, where $\left|\mathcal{T}^{0}\right|=j$ and $\left|\mathcal{T}^{\infty}\right|=2 j+1$. Let us denote the collection of trees in the $j$ th generation (i.e. with $j$ parental nodes) by $T(j)$, i.e.

$$
T(j):=\{\mathcal{T}: \mathcal{T} \text { is a tree with }|\mathcal{T}|=3 j+1\} .
$$

Now, we introduce the notion of ordered trees.

Definition 3.3. We say that a sequence $\left\{\mathcal{T}_{j}\right\}_{j=1}^{J}$ is a chronicle of $J$ generations, if

(i) $\mathcal{T}_{j} \in T(j)$ for each $j=1, \ldots, J$,

(ii) $\mathcal{T}_{j+1}$ is obtained by changing one of the terminal nodes in $\mathcal{T}_{j}$ into a non-terminal node (with three children), $j=1, \ldots, J-1$.

Given a chronicle $\left\{\mathcal{T}_{j}\right\}_{j=1}^{J}$ of $J$ generations, we refer to $\mathcal{T}_{J}$ as an ordered tree of the $J$ th generation. We denote the collection of the ordered trees of the $J$ th generation by $\mathfrak{T}(J)$. Note that the cardinality of $\mathfrak{T}(J)$ is given by

$$
|\mathfrak{T}(J)|=1 \cdot 3 \cdot 5 \cdots \cdot(2 J-1)=: c_{J} .
$$

Remark 3.4. Given two ordered trees $\mathcal{T}_{J}$ and $\widetilde{\mathcal{T}}_{J}$ of the $J$ th generation, it may happen that $\mathcal{T}_{J}=\widetilde{\mathcal{T}}_{J}$ as trees (namely as graphs) according to Definition [3.2, while $\mathcal{T}_{J} \neq \widetilde{\mathcal{T}}_{J}$ as ordered trees according to Definition 3.3. Namely, the notion of ordered trees comes with associated chronicles; it encodes not only the shape of a tree but also how it "grew". Henceforth, when we refer to an ordered tree $\mathcal{T}_{J}$ of the $J$ th generation, it is understood that there is an underlying chronicle $\left\{\mathcal{T}_{j}\right\}_{j=1}^{J}$.

Definition 3.5. Given an ordered tree $\mathcal{T}$ (of the $J$ th generation for some $J \in \mathbb{N}$ ), we define an index function $\mathbf{n}: \mathcal{T} \rightarrow \mathbb{Z}$ such that,

(i) $n_{a}=n_{a_{1}}-n_{a_{2}}+n_{a_{3}}$ for $a \in \mathcal{T}^{0}$, where $a_{1}, a_{2}$, and $a_{3}$ denote the children of $a$, 
(ii) $\left\{n_{a}, n_{a_{2}}\right\} \cap\left\{n_{a_{1}}, n_{a_{3}}\right\}=\emptyset$ for $a \in \mathcal{T}^{0}$,

(iii) $\left|\mu_{1}\right|:=\left|2\left(n_{r}-n_{r_{1}}\right)\left(n_{r}-n_{r_{3}}\right)\right|>N$, where $r$ is the root node, (recall that we are on $A_{N}^{c}$ - see (2.5)

where we identified $\mathbf{n}: \mathcal{T} \rightarrow \mathbb{Z}$ with $\left\{n_{a}\right\}_{a \in \mathcal{T}} \in \mathbb{Z}^{\mathcal{T}}$.

We use $\mathfrak{N}(\mathcal{T}) \subset \mathbb{Z}^{\mathcal{T}}$ to denote the collection of such index functions $\mathbf{n}$.

Remark 3.6. Note that $\mathbf{n}=\left\{n_{a}\right\}_{a \in \mathcal{T}}$ is completely determined once we specify the values $n_{a}$ for $a \in \mathcal{T}^{\infty}$.

Given an ordered tree $\mathcal{T}_{J}$ of the $J$ th generation with the chronicle $\left\{\mathcal{T}_{j}\right\}_{j=1}^{J}$ and associated index functions $\mathbf{n} \in \mathfrak{N}\left(\mathcal{T}_{J}\right)$, we would like to keep track of the "generations" of frequencies. In the following, we use superscripts to denote such generations of frequencies.

Fix $\mathbf{n} \in \mathfrak{N}\left(\mathcal{T}_{J}\right)$. Consider $\mathcal{T}_{1}$ of the first generation. Its nodes consist of the root node $r$ and its children $r_{1}, r_{2}$, and $r_{3}$. We define the first generation of frequencies by

$$
\left(n^{(1)}, n_{1}^{(1)}, n_{2}^{(1)}, n_{3}^{(1)}\right):=\left(n_{r}, n_{r_{1}}, n_{r_{2}}, n_{r_{3}}\right) .
$$

From Definition 3.5, we have

$$
n^{(1)}=n_{1}^{(1)}-n_{2}^{(1)}+n_{3}^{(1)}, \quad n_{2}^{(1)} \neq n_{1}^{(1)}, n_{3}^{(1)} .
$$

The ordered tree $\mathcal{T}_{2}$ of the second generation is obtained from $\mathcal{T}_{1}$ by changing one of its terminal nodes $a=r_{k} \in \mathcal{T}_{1}^{\infty}$ for some $k \in\{1,2,3\}$ into a non-terminal node. Then, we define the second generation of frequencies by

$$
\left(n^{(2)}, n_{1}^{(2)}, n_{2}^{(2)}, n_{3}^{(2)}\right):=\left(n_{a}, n_{a_{1}}, n_{a_{2}}, n_{a_{3}}\right) .
$$

Then, we have $n^{(2)}=n_{k}^{(1)}$ for some $k \in\{1,2,3\}$,

$$
n^{(2)}=n_{1}^{(2)}-n_{2}^{(2)}+n_{3}^{(2)}, \quad n_{2}^{(2)} \neq n_{1}^{(2)}, n_{3}^{(2)},
$$

where the last identities follow from Definition 3.5.

As we see later, this corresponds to introducing a new set of frequencies after the first differentiation by parts. For example, in (3.1), we assumed that the time derivative falls on $v_{n_{1}^{(1)}}$. This corresponds to changing the "first" child $r_{1} \in \mathcal{T}_{1}^{\infty}$ into a non-terminal node, and we have

$$
\left(n^{(2)}, n_{1}^{(2)}, n_{2}^{(2)}, n_{3}^{(2)}\right):=\left(n_{1}, m_{1}, m_{2}, m_{3}\right)
$$

After $j-1$ steps, the ordered tree $\mathcal{T}_{j}$ of the $j$ th generation is obtained from $\mathcal{T}_{j-1}$ by changing one of its terminal nodes $a \in \mathcal{T}_{j-1}^{\infty}$ into a non-terminal node. Then, we define the $j$ th generation of frequencies by

$$
\left(n^{(j)}, n_{1}^{(j)}, n_{2}^{(j)}, n_{3}^{(j)}\right):=\left(n_{a}, n_{a_{1}}, n_{a_{2}}, n_{a_{3}}\right) .
$$

As before, from Definition 3.5, we have

$$
n^{(j)}=n_{1}^{(j)}-n_{2}^{(j)}+n_{3}^{(j)}, \quad n_{2}^{(j)} \neq n_{1}^{(j)}, n_{3}^{(j)} .
$$

Also, we have $n^{(j)}=n_{k}^{(m)}\left(=n_{a}\right)$ for some $m \in\{1, \ldots, j-1\}$ and $k \in\{1,2,3\}$, since this corresponds to the frequency of some terminal node in $\mathcal{T}_{j-1}$. 
In the following, we pictorially present an example of an ordered tree $\mathcal{T} \in \mathfrak{T}(4)$ with $\mathbf{n} \in \mathfrak{N}(\mathcal{T}):$

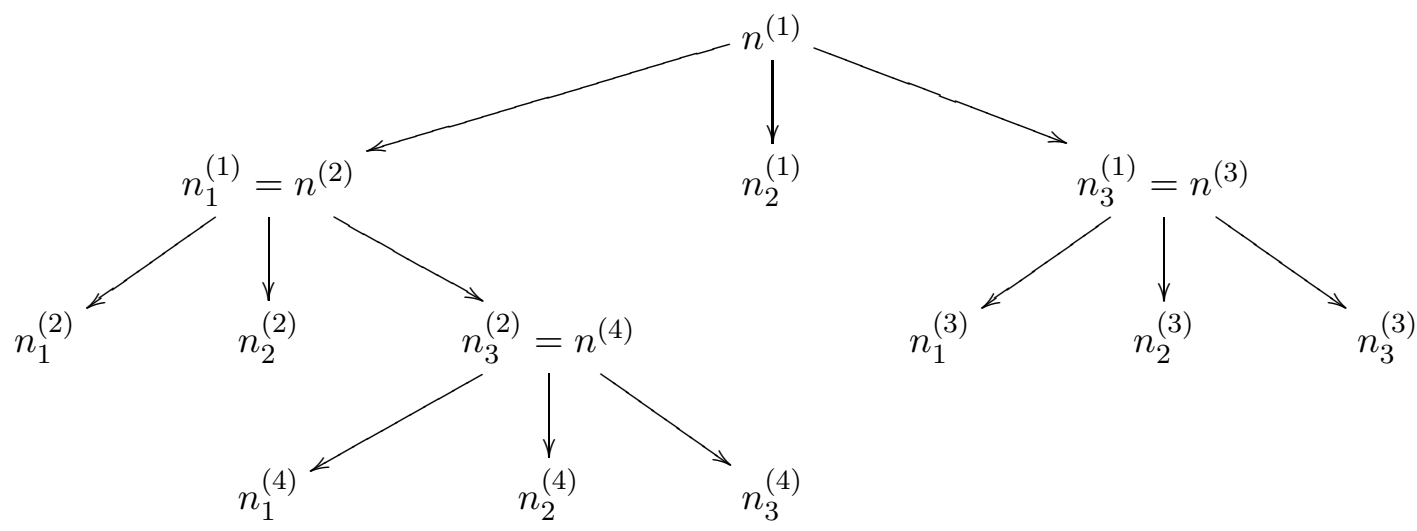

Here, we have ornamented the nodes with the values of $\mathbf{n}=\left\{n_{a}\right\}_{a \in \mathcal{T}} \in \mathfrak{N}(\mathcal{T})$, specifying the generations of frequencies as discussed above.

We use $\mu_{j}$ to denote the corresponding phase factor introduced at the $j$ th generation. Namely, we have

$$
\begin{aligned}
\mu_{j} & =\mu_{j}\left(n^{(j)}, n_{1}^{(j)}, n_{2}^{(j)}, n_{3}^{(j)}\right):=\left(n^{(j)}\right)^{2}-\left(n_{1}^{(j)}\right)^{2}+\left(n_{2}^{(j)}\right)^{2}-\left(n_{3}^{(j)}\right)^{2} \\
& =2\left(n_{2}^{(j)}-n_{1}^{(j)}\right)\left(n_{2}^{(j)}-n_{3}^{(j)}\right)=2\left(n^{(j)}-n_{1}^{(j)}\right)\left(n^{(j)}-n_{3}^{(j)}\right),
\end{aligned}
$$

where the last two equalities hold thanks to (3.3).

Lastly, for a fixed ordered tree $\mathcal{T}$, we denote by $B_{j}=B_{j}(\mathcal{T})$ the set of all possible frequencies in the $j$ th generation.

3.2. Example: second and third generations. Using these notations, we can rewrite $\mathcal{N}_{3}$ in (3.1) as

$$
\mathcal{N}^{(2)}(n):=\left(\mathcal{N}_{3}\right)_{n}=\sum_{\mathcal{T}_{2} \in \mathfrak{T}(2)} \sum_{\substack{\mathbf{n} \in \mathfrak{N}\left(\mathcal{T}_{2}\right) \\ \mathbf{n}_{r}=n}} \frac{e^{-i\left(\mu_{1}+\mu_{2}\right) t}}{\mu_{1}} \prod_{a \in \mathcal{T}_{2}^{\infty}} v_{n_{a}} .
$$

Here, we included the contribution of a similar term arising from $\mathcal{N}_{222}$ in (2.13), i.e. when the time derivative falls on the second factor $v_{n_{2}} 7$ Strictly speaking, the new phase factor may be $\mu_{1}-\mu_{2}$ when the time derivative falls on the complex conjugate. However, for our analysis, it makes no difference and hence we simply write it as $\mu_{1}+\mu_{2}$. The same comments apply in the following. Also, recall that the set of frequencies are restricted onto $A_{N}^{c}$ defined in (2.5). See Definition 3.5 (iii). In the following, similar restrictions on $\mu_{j}$ appear, but we suppress such restrictions for simplicity of notations, when it is clear from the context.

Next, we divide the Fourier space into

$$
C_{1}=\left\{\left|\mu_{1}+\mu_{2}\right| \lesssim 5^{3}\left|\mu_{1}\right|^{1-\frac{1}{100}}\right\}
$$

\footnotetext{
${ }^{7}$ As before, we only keep track of the absolute values of coefficients in the following. We may also drop the minus signs and the complex number $i$.
} 
and its complement $C_{1}^{c} 8$ Then, write

$$
\mathcal{N}^{(2)}=\mathcal{N}_{1}^{(2)}+\mathcal{N}_{2}^{(2)}
$$

where $\mathcal{N}_{1}^{(2)}$ is the restriction of $\mathcal{N}^{(2)}$ onto $C_{1}$ and $\mathcal{N}_{2}^{(2)}:=\mathcal{N}^{(2)}-\mathcal{N}_{1}^{(2)}$.

Lemma 3.7. Let $\mathcal{N}_{1}^{(2)}$ be as in (3.7). Then, we have

$$
\begin{aligned}
\left\|\mathcal{N}_{1}^{(2)}(v)\right\|_{L^{2}} & \lesssim N^{-\frac{1}{200}+}\|v\|_{L^{2}}^{5} \\
\left\|\mathcal{N}_{1}^{(2)}(v)-\mathcal{N}_{1}^{(2)}(w)\right\|_{L^{2}} & \lesssim N^{-\frac{1}{200}+}\left(\|v\|_{L^{2}}^{4}+\|w\|_{L^{2}}^{4}\right)\|v-w\|_{L^{2}}
\end{aligned}
$$

Proof. We only prove (3.8) since (3.9) follows in a similar manner. Since we are on $A_{N}^{c}$ (see (2.5)), we have $\left|\mu_{1}\right|>N$. Next, we use the divisor counting argument as in the proof of Lemma 2.2. It follows from (2.6) that for fixed $n$ and $\mu_{1}$, there are at most $o\left(\left|\mu_{1}\right|^{0+}\right)$ many choices for $n_{1}^{(1)}$ and $n_{3}^{(1)}$ on $B_{1}$ (and hence for $n_{2}^{(1)}$ from $n^{(1)}=n_{1}^{(1)}-n_{2}^{(1)}+n_{3}^{(1)}$ ). Similarly, for fixed $n^{(2)}=n_{1}^{(1)}$ and $\mu_{2}$, there are at most $o\left(\left|\mu_{2}\right|^{0+}\right)$ many choices for $n_{1}^{(2)}, n_{2}^{(2)}$, and $n_{3}^{(2)}$ on $B_{2}$.

The main point is to control $\left|\mu_{2}\right|$ in terms of $\left|\mu_{1}\right|$. From (3.6), we have $\left|\mu_{2}\right| \sim\left|\mu_{1}\right|$. Moreover, for fixed $\left|\mu_{1}\right|$, there are at most $O\left(\left|\mu_{1}\right|^{1-\frac{1}{100}}\right)$ many choices for $\mu_{2}$. Hence, by Cauchy-Schwarz inequality, we have

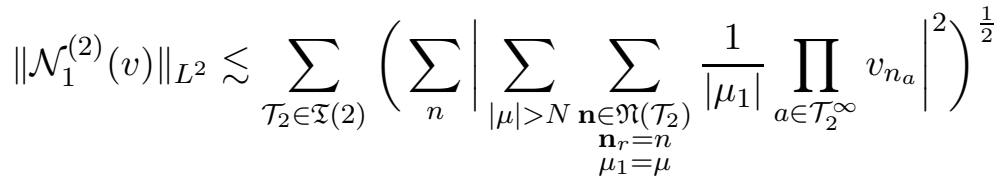

$$
\begin{aligned}
& \lesssim\left\{\sum_{n}\left(\sum_{|\mu|>N} \frac{1}{|\mu|^{2}}|\mu|^{1-\frac{1}{100}+}\right)\left(\sum_{\substack{\mathbf{n} \in \mathfrak{N}\left(\mathcal{T}_{2}\right) \\
\mathbf{n}_{r}=n}} \prod_{a \in \mathcal{T}_{2}^{\infty}}\left|v_{n_{a}}\right|^{2}\right)\right\}^{\frac{1}{2}} \\
& \leq N^{-\frac{1}{200}+}\|v\|_{L^{2}}^{5} .
\end{aligned}
$$

Next, we apply (the second step of) Poincaré-Dulac normal form reduction to $\mathcal{N}_{2}^{(2)}$. Note that we have

$$
\left|\mu_{1}+\mu_{2}\right| \gg 5^{3}\left|\mu_{1}\right|^{1-\frac{1}{100}}>5^{3} N^{1-\frac{1}{100}}
$$

\footnotetext{
${ }^{8}$ Clearly, the number $5^{3}$ in (3.6) does not make any difference at this point. However, we insert it to match with (3.33). See also (3.17) and (3.23).
} 
on the support of $\mathcal{N}_{2}^{(2)}$, i.e. on $C_{1}^{c}$. After differentiation by parts, we obtain

$$
\begin{aligned}
\mathcal{N}_{2}^{(2)}(n)= & \partial_{t}\left[\sum_{\mathcal{T}_{2} \in \mathfrak{T}(2)} \sum_{\substack{\mathbf{n} \in \mathfrak{N}\left(\mathcal{T}_{2}\right) \\
\mathbf{n}_{r}=n}} \frac{e^{-i\left(\mu_{1}+\mu_{2}\right) t}}{\mu_{1}\left(\mu_{1}+\mu_{2}\right)} \prod_{a \in \mathcal{T}_{2}^{\infty}} v_{n_{a}}\right] \\
& -\sum_{\mathcal{T}_{2} \in \mathfrak{T}(2)} \sum_{\substack{\mathbf{n} \in \mathfrak{N}\left(\mathcal{T}_{2}\right) \\
\mathbf{n}_{r}=n}} \frac{e^{-i\left(\mu_{1}+\mu_{2}\right) t}}{\mu_{1}\left(\mu_{1}+\mu_{2}\right)} \partial_{t}\left(\prod_{a \in \mathcal{T}_{2}^{\infty}} v_{n_{a}}\right) \\
= & \partial_{t}\left[\sum_{\mathcal{T}_{2} \in \mathfrak{T}(2)} \sum_{\substack{\mathbf{n} \in \mathfrak{N}\left(\mathcal{T}_{2}\right) \\
\mathbf{n}_{r}=n}} \frac{e^{-i\left(\mu_{1}+\mu_{2}\right) t}}{\mu_{1}\left(\mu_{1}+\mu_{2}\right)} \prod_{a \in \mathcal{T}_{2}^{\infty}} v_{n_{a}}\right] \\
& -\sum_{\mathcal{T}_{2} \in \mathfrak{T}(2)} \sum_{\substack{b \in \mathcal{T}_{2}^{\infty} \\
\mathbf{n} \in \mathfrak{N}\left(\mathcal{T}_{2}\right)}} \frac{e^{-i\left(\mu_{1}+\mu_{2}\right) t}}{\mu_{1}\left(\mu_{1}+\mu_{2}\right)} \partial_{t} v_{n_{b}} \prod_{a \in \mathcal{T}_{2}^{\infty} \backslash\{b\}} v_{n_{a}} \\
= & \partial_{t}\left[\sum_{\mathcal{T}_{2} \in \mathfrak{T}(2)} \sum_{\substack{\mathbf{n} \in \mathfrak{N}\left(\mathcal{T}_{2}\right) \\
\mathbf{n}_{r}=n}} \frac{e^{-i\left(\mu_{1}+\mu_{2}\right) t}}{\mu_{1}\left(\mu_{1}+\mu_{2}\right)} \prod_{a \in \mathcal{T}_{2}^{\infty}} v_{n_{a}}\right] \\
& -\sum_{\mathcal{T}_{2} \in \mathfrak{T}(2)} \sum_{b \in \mathcal{T}_{2}^{\infty} \mathbf{n} \in \mathfrak{n}\left(\mathcal{T}_{2}\right)} \sum_{\mathbf{n}_{r}=n} \frac{e^{-i\left(\mu_{1}+\mu_{2}\right) t}}{\mu_{1}\left(\mu_{1}+\mu_{2}\right)}\left(\mathcal{R}_{1}-\mathcal{R}_{2}\right)_{n_{b}} \prod_{a \in \mathcal{T}_{2}^{\infty} \backslash\{b\}} v_{n_{a}} \\
& -\sum_{\mathcal{T}_{3} \in \mathfrak{T}(3)} \sum_{\substack{\mathbf{n} \in \mathfrak{N}\left(\mathcal{T}_{3}\right) \\
\mathbf{n}_{r}=n}} \frac{e^{-i\left(\mu_{1}+\mu_{2}+\mu_{3}\right) t}}{\mu_{t}\left(\mu_{1}+\mu_{2}\right)} \prod_{a \in \mathcal{T}_{3}^{\infty}} v_{n_{a}} \\
= & \partial_{t} \mathcal{N}_{0}^{(3)}(n)+\mathcal{N}_{r}^{(3)}(n)+\mathcal{N}^{(3)}(n) .
\end{aligned}
$$

In the third equality, we used (2.1) and replaced $\partial_{t} v_{n_{b}}$ by the resonant part $\left(\mathcal{R}_{1}-\mathcal{R}_{2}\right)\left(n_{b}\right)$ and the non-resonant part $\mathcal{N}_{1}\left(n_{b}\right)$. As for the contribution from the non-resonant part, we replace the frequency $n_{b}$ by $n_{b_{1}}, n_{b_{2}}$, and $n_{b_{3}}$ such that $n_{b}=n_{b_{1}}-n_{b_{2}}+n_{b_{3}}$ and $n_{b_{2}} \neq n_{b_{1}}, n_{b_{3}}$, which corresponds to extending the tree $\mathcal{T}_{2} \in \mathfrak{T}(2)$ (and $\mathbf{n} \in \mathfrak{N}\left(\mathcal{T}_{2}\right)$ ) to $\mathcal{T}_{3} \in \mathfrak{T}(3)$ (and to $\mathbf{n} \in \mathfrak{N}\left(\mathcal{T}_{3}\right)$, respectively) by replacing the terminal node $b \in \mathcal{T}_{2}^{\infty}$ into a non-terminal node with three children $b_{1}, b_{2}$, and $b_{3}$.

First, let us estimate the easier terms $\mathcal{N}_{0}^{(3)}$ and $\mathcal{N}_{r}^{(3)}$.

Lemma 3.8. Let $\mathcal{N}_{0}^{(3)}$ be as in (3.11). Then, we have

$$
\begin{aligned}
\left\|\mathcal{N}_{0}^{(3)}(v)\right\|_{L^{2}} & \lesssim N^{-1+\frac{1}{200}+}\|v\|_{L^{2}}^{5} \\
\left\|\mathcal{N}_{0}^{(3)}(v)-\mathcal{N}_{0}^{(3)}(w)\right\|_{L^{2}} & \lesssim N^{-1+\frac{1}{200}+}\left(\|v\|_{L^{2}}^{4}+\|w\|_{L^{2}}^{4}\right)\|v-w\|_{L^{2}} .
\end{aligned}
$$

Proof. We only prove (3.12) since (3.13) follows in a similar manner. As in the proof of Lemma 3.7, it follows from (2.6) that for fixed $n$ and $\mu_{1}$, there are at most $o\left(\left|\mu_{1}\right|^{0+}\right)$ many choices for $n_{1}^{(1)}, n_{2}^{(1)}$, and $n_{3}^{(1)}$ on $B_{1}$. Similarly, for fixed $n^{(2)}=n_{1}^{(1)}$ and $\mu_{2}$, there are at most $o\left(\left|\mu_{2}\right|^{0+}\right)$ many choices for $n_{1}^{(2)}, n_{2}^{(2)}$, and $n_{3}^{(2)}$ on $B_{2}$. 
With $\widetilde{\mu}_{2}=\mu_{1}+\mu_{2}$, we have $\left|\mu_{2}\right| \lesssim \max \left(\left|\mu_{1}\right|,\left|\widetilde{\mu}_{2}\right|\right)$. Then, by Cauchy-Schwarz inequality, we have

$$
\begin{aligned}
\left\|\mathcal{N}_{0}^{(3)}(v)\right\|_{L^{2}} \lesssim \sum_{\mathcal{T}_{2} \in \mathfrak{T}(2)}\left\{\sum_{n}\left(\sum_{\substack{\left|\mu_{1}\right|>N \\
\left|\widetilde{\mu}_{2}\right|>5^{3} N^{1-\frac{1}{100}}}} \frac{1}{\left|\mu_{1}\right|^{2}\left|\widetilde{\mu}_{2}\right|^{2}}\left|\mu_{1}\right|^{0+}\left|\mu_{2}\right|^{0+}\right)\right. \\
\left.\quad \times\left(\sum_{\substack{\mathbf{n} \in \mathfrak{N}\left(\mathcal{T}_{2}\right) \\
\mathbf{n}_{r}=n}} \prod_{a \in \mathcal{T}_{2}^{\infty}}\left|v_{n_{a}}\right|^{2}\right)\right\}^{\frac{1}{2}} \\
\lesssim N^{-1+\frac{1}{200}+}\|v\|_{L^{2}}^{5} .
\end{aligned}
$$

Lemma 3.9. Let $\mathcal{N}_{r}^{(3)}$ be as in (3.11). Then, we have

$$
\begin{aligned}
\left\|\mathcal{N}_{r}^{(3)}(v)\right\|_{L^{2}} & \lesssim N^{-1+\frac{1}{200}+}\|v\|_{L^{2}}^{7} \\
\left\|\mathcal{N}_{r}^{(3)}(v)-\mathcal{N}_{r}^{(3)}(w)\right\|_{L^{2}} & \lesssim N^{-1+\frac{1}{200}+}\left(\|v\|_{L^{2}}^{6}+\|w\|_{L^{2}}^{6}\right)\|v-w\|_{L^{2}} .
\end{aligned}
$$

Proof. This lemma follows from Lemmata 3.8 and 2.1. Note that, given $\mathcal{T}_{2} \in \mathfrak{T}(2)$, we have $\#\left\{b: b \in \mathcal{T}_{2}\right\}=5$.

Now, we treat $\mathcal{N}^{(3)}$. As before, we write

$$
\mathcal{N}^{(3)}=\mathcal{N}_{1}^{(3)}+\mathcal{N}_{2}^{(3)}
$$

where $\mathcal{N}_{1}^{(3)}$ is the restriction of $\mathcal{N}^{(3)}$ onto

$$
C_{2}=\left\{\left|\widetilde{\mu}_{3}\right| \lesssim 7^{3}\left|\widetilde{\mu}_{2}\right|^{1-\frac{1}{100}}\right\} \cup\left\{\left|\widetilde{\mu}_{3}\right| \lesssim 7^{3}\left|\mu_{1}\right|^{1-\frac{1}{100}}\right\}
$$

where $\widetilde{\mu}_{2}:=\mu_{1}+\mu_{2}$ and $\widetilde{\mu}_{3}:=\mu_{1}+\mu_{2}+\mu_{3}$, and $\mathcal{N}_{2}^{(3)}:=\mathcal{N}^{(3)}-\mathcal{N}_{1}^{(3)}$.

Lemma 3.10. Let $\mathcal{N}_{1}^{(3)}$ be as in (3.16). Then, we have

$$
\begin{aligned}
\left\|\mathcal{N}_{1}^{(3)}(v)\right\|_{L^{2}} & \lesssim N^{-\frac{1}{2}+}\|v\|_{L^{2}}^{7}, \\
\left\|\mathcal{N}_{1}^{(3)}(v)-\mathcal{N}_{1}^{(3)}(w)\right\|_{L^{2}} & \lesssim N^{-\frac{1}{2}+}\left(\|v\|_{L^{2}}^{6}+\|w\|_{L^{2}}^{6}\right)\|v-w\|_{L^{2}} .
\end{aligned}
$$

Proof. We only prove (3.18) since (3.19) follows in a similar manner. The proof is very similar to that of Lemma 3.7, i.e. we use the divisor counting argument. It follows from (2.6) that for fixed $n$ and $\mu_{1}$, there are at most $o\left(\left|\mu_{1}\right|^{0+}\right)$ many choices for $n_{1}^{(1)}, n_{2}^{(1)}$, and $n_{3}^{(1)}$ on $B_{1}$. For fixed $n^{(2)}=n_{1}^{(1)}$ and $\mu_{2}$, there are at most $o\left(\left|\mu_{2}\right|^{0+}\right)$ many choices for $n_{1}^{(2)}$, $n_{2}^{(2)}$, and $n_{3}^{(2)}$ on $B_{2}$. Moreover, for fixed $n^{(3)}=n_{1}^{(2)}$ and $\mu_{3}$, there are at most $o\left(\left|\mu_{3}\right|^{0+}\right)$ many choices for $n_{1}^{(3)}, n_{2}^{(3)}$, and $n_{3}^{(3)}$ on $B_{3}$.

First, we assume that $\left|\widetilde{\mu}_{3}\right| \lesssim\left|\widetilde{\mu}_{2}\right|^{1-\frac{1}{100}}$ holds in (3.17). Then, we have $\left|\mu_{3}\right| \sim\left|\widetilde{\mu}_{2}\right|$ since $\widetilde{\mu}_{3}=\widetilde{\mu}_{2}+\mu_{3}$. Moreover, for fixed $\left|\widetilde{\mu}_{2}\right|$, namely for fixed $\mu_{1}$ and $\mu_{2}$, there are at most $O\left(\left|\widetilde{\mu}_{2}\right|^{1-\frac{1}{100}}\right)$ many choices for $\widetilde{\mu}_{3}$, and hence for $\mu_{3}=\widetilde{\mu}_{3}-\widetilde{\mu}_{2}$. Also, we have $\left|\mu_{2}\right| \lesssim$ 
$\max \left(\left|\mu_{1}\right|,\left|\widetilde{\mu}_{2}\right|\right)$ and (3.10). Then, by Cauchy-Schwarz inequality, we have

$$
\begin{aligned}
\left\|\mathcal{N}_{1}^{(3)}(v)\right\|_{L^{2}} & \lesssim \sum_{\mathcal{T}_{3} \in \mathfrak{T}(3)}\left(\sum_{n}\left|\sum_{\substack{\mathbf{n} \in \mathfrak{N}\left(\mathcal{T}_{3}\right) \\
\mathbf{n}_{r}=n}} \frac{1}{\left|\mu_{1}\right|\left|\widetilde{\mu}_{2}\right|} \prod_{a \in \mathcal{T}_{3}^{\infty}} v_{n_{a}}\right|^{2}\right)^{\frac{1}{2}} \\
& \lesssim\left\{\sum_{n}\left(\sum_{\substack{\left|\mu_{1}\right|>N \\
\left|\widetilde{\mu}_{2}\right|>5^{3} N^{1-\frac{1}{100}}}} \frac{1}{\left|\mu_{1}\right|^{2}\left|\widetilde{\mu}_{2}\right|^{2}}\left|\mu_{1}\right|^{0+}\left|\mu_{2}\right|^{0+}\left|\mu_{3}\right|^{0+}\left|\widetilde{\mu}_{2}\right|^{1-\frac{1}{100}}\right)\right. \\
& \left.\times\left(\sum_{\substack{\mathbf{n} \in \mathfrak{N}\left(\mathcal{T}_{3}\right) \\
\mathbf{n}_{r}=n}} \prod_{a \in \mathcal{T}_{3}^{\infty}}\left|v_{n_{a}}\right|^{2}\right)\right\}^{\frac{1}{2}} \\
& \lesssim\left\{\sum_{n}\left(\sum_{\substack{\left|\mu_{1}\right|>N \\
\left|\widetilde{\mu}_{2}\right|>5^{3} N^{1-\frac{1}{100}}}} \frac{1}{\left|\mu_{1}\right|^{2-}\left|\widetilde{\mu}_{2}\right|^{1+\frac{1}{100}-}}\right)\left(\sum_{\substack{\mathbf{n} \in \mathfrak{N}\left(\mathcal{T}_{3}\right) \\
\mathbf{n}_{r}=n}} \prod_{a \in \mathcal{T}_{3}^{\infty}}\left|v_{n_{a}}\right|^{2}\right)\right\}^{\frac{1}{2}} \\
& \lesssim N^{-\frac{1}{2}\|v\|_{L^{2}}^{7} .}
\end{aligned}
$$

If $\left|\widetilde{\mu}_{3}\right| \lesssim\left|\mu_{1}\right|^{1-\frac{1}{100}}$ holds in (3.17), then, for fixed $\mu_{1}$ and $\mu_{2}$, there are at most $O\left(\left|\mu_{1}\right|^{1-\frac{1}{100}}\right)$ many choices for $\widetilde{\mu}_{3}$, and hence for $\mu_{3}$. By repeating the same computation, we obtain $\left|\mu_{1}\right|^{-1-\frac{1}{100}+}\left|\widetilde{\mu}_{2}\right|^{-2+}$ in (3.20), yielding (3.18) with $N^{-\frac{1}{2}+}$.

Next, we apply (the third step of) Poincaré-Dulac normal form reduction to $\mathcal{N}_{2}^{(3)}$. Note that we have

$$
\left|\widetilde{\mu}_{3}\right|=\left|\mu_{1}+\mu_{2}+\mu_{3}\right| \gg 7^{3}\left|\mu_{1}\right|^{1-\frac{1}{100}}>7^{3} N^{1-\frac{1}{100}}
$$

on the support of $\mathcal{N}_{2}^{(3)}$, i.e. on $C_{2}^{c}$. After differentiation by parts, we obtain

$$
\begin{aligned}
\mathcal{N}_{2}^{(3)}(n)= & \partial_{t}\left[-\sum_{\mathcal{T}_{3} \in \mathfrak{T}(3)} \sum_{\substack{\mathbf{n} \in \mathfrak{N}\left(\mathcal{T}_{3}\right) \\
\mathbf{n}_{r}=n}} \frac{e^{-i\left(\mu_{1}+\mu_{2}+\mu_{3}\right) t}}{\mu_{1}\left(\mu_{1}+\mu_{2}\right)\left(\mu_{1}+\mu_{2}+\mu_{3}\right)} \prod_{a \in \mathcal{T}_{3}^{\infty}} v_{n_{a}}\right] \\
& +\sum_{\mathcal{T}_{3} \in \mathfrak{T}(3)} \sum_{\substack{b \in \mathcal{T}_{3}^{\infty} \\
\sum_{\mathbf{n} \in \mathfrak{N}\left(\mathcal{T}_{3}\right)}}} \frac{e^{-i\left(\mu_{1}+\mu_{2}+\mu_{3}\right) t}}{\mu_{1}\left(\mu_{1}+\mu_{2}\right)\left(\mu_{1}+\mu_{2}+\mu_{3}\right)}\left(\mathcal{R}_{1}-\mathcal{R}_{2}\right)_{n_{b}} \prod_{a \in \mathcal{T}_{3}^{\infty} \backslash\{b\}} v_{n_{a}} \\
& +\sum_{\mathcal{T}_{4} \in \mathfrak{T}(4)} \sum_{\substack{\mathbf{n} \in \mathfrak{N}\left(\mathcal{T}_{4}\right) \\
\mathbf{n} r=n}} \frac{e^{-i\left(\mu_{1}+\mu_{2}+\mu_{3}+\mu_{4}\right) t}}{\mu_{1}\left(\mu_{1}+\mu_{2}\right)\left(\mu_{1}+\mu_{2}+\mu_{3}\right)} \prod_{a \in \mathcal{T}_{4}^{\infty}} v_{n_{a}} \\
= & : \partial_{t} \mathcal{N}_{0}^{(4)}(n)+\mathcal{N}_{r}^{(4)}(n)+\mathcal{N}^{(4)}(n) .
\end{aligned}
$$

We can clearly estimate $\mathcal{N}_{0}^{(4)}$ and $\mathcal{N}_{r}^{(4)}$, with $\left|\mu_{1}\right|>N$, (3.10), and (3.21), just as in Lemmata 3.8 and 3.9. As for $\mathcal{N}^{(4)}$, we can write

$$
\mathcal{N}^{(4)}=\mathcal{N}_{1}^{(4)}+\mathcal{N}_{2}^{(4)}
$$

as the restrictions onto

$$
C_{3}=\left\{\left|\widetilde{\mu}_{4}\right| \lesssim 9^{3}\left|\widetilde{\mu}_{3}\right|^{1-\frac{1}{100}}\right\} \cup\left\{\left|\widetilde{\mu}_{4}\right| \lesssim 9^{3}\left|\mu_{1}\right|^{1-\frac{1}{100}}\right\},
$$


where $\widetilde{\mu}_{4}:=\mu_{1}+\mu_{2}+\mu_{3}+\mu_{4}$, and its complement $C_{3}^{c}$, respectively. Then, $\mathcal{N}_{1}^{(4)}$ can be estimated as in Lemma 3.10 and we can apply (the fourth step of) Poincaré-Dulac normal form reduction to $\mathcal{N}_{2}^{(4)}$. In this way, we iterate Poincaré-Dulac normal form reductions.

3.3. General step: $J$ th generation. After the $J$ th step, we have

$$
\begin{aligned}
\mathcal{N}_{2}^{(J)}(n)= & \partial_{t}\left[\mp \sum_{\mathcal{T}_{J} \in \mathfrak{T}(J)} \sum_{\substack{\mathbf{n} \in \mathfrak{N}\left(\mathcal{T}_{J}\right) \\
\mathbf{n}_{r}=n}} \frac{e^{-i \widetilde{\mu}_{J} t}}{\widehat{\mu}_{J}} \prod_{a \in \mathcal{T}_{J}^{\infty}} v_{n_{a}}\right] \\
& \pm \sum_{\mathcal{T}_{J} \in \mathfrak{T}(J)} \sum_{b \in \mathcal{T}_{J}^{\infty}} \sum_{\substack{\mathbf{n} \in \mathfrak{N}\left(\mathcal{T}_{J}\right) \\
\mathbf{n}_{r}=n}} \frac{e^{-i \widetilde{\mu}_{J} t}}{\widehat{\mu}_{J}}\left(\mathcal{R}_{1}-\mathcal{R}_{2}\right)_{n_{b}} \prod_{a \in \mathcal{T}_{J}^{\infty} \backslash\{b\}} v_{n_{a}} \\
& \pm \sum_{\mathcal{T}_{J+1} \in \mathfrak{T}(J+1)} \sum_{\mathbf{n} \in \mathfrak{N}_{\mathbf{N}_{r}\left(\mathcal{T}_{J+1}\right)}} \frac{e^{-i \widetilde{\mu}_{J+1} t}}{\widehat{\mu}_{J}} \prod_{a \in \mathcal{T}_{J+1}^{\infty}} v_{n_{a}} \\
= & : \partial_{t} \mathcal{N}_{0}^{(J+1)}(n)+\mathcal{N}_{r}^{(J+1)}(n)+\mathcal{N}^{(J+1)}(n),
\end{aligned}
$$

where $\widetilde{\mu}_{J}$ and $\widehat{\mu}_{J}$ are given by

$$
\widetilde{\mu}_{J}:=\sum_{j=1}^{J} \mu_{j}, \quad \text { and } \quad \widehat{\mu}_{J}:=\prod_{j=1}^{J} \widetilde{\mu}_{j} .
$$

Keep in mind that $\left|\mu_{1}\right|>N$ and

$$
\left|\widetilde{\mu}_{j}\right| \gg(2 j+1)^{3} \max \left(\left|\widetilde{\mu}_{j-1}\right|^{1-\frac{1}{100}},\left|\mu_{1}\right|^{1-\frac{1}{100}}\right)>(2 j+1)^{3} N^{1-\frac{1}{100}},
$$

for $j=2, \ldots, J$. First, we estimate $\mathcal{N}_{0}^{(J+1)}$ and $\mathcal{N}_{r}^{(J+1)}$.

Lemma 3.11. Let $\mathcal{N}_{0}^{(J+1)}$ be as in (3.24). Then, we have9

$$
\begin{aligned}
&\left\|\mathcal{N}_{0}^{(J+1)}(v)\right\|_{L^{2}} \lesssim N^{-\frac{J}{2}+\frac{(J-1)}{200}+\|v\|_{L^{2}}^{2 J+1}} \\
&\left\|\mathcal{N}_{0}^{(J+1)}(v)-\mathcal{N}_{0}^{(J+1)}(w)\right\|_{L^{2}} \lesssim N^{-\frac{J}{2}+\frac{(J-1)}{200}+}\left(\|v\|_{L^{2}}^{2 J}+\|w\|_{L^{2}}^{2 J}\right)\|v-w\|_{L^{2}}
\end{aligned}
$$

Proof. We only prove (3.26) since (3.27) follows in a similar manner. Note that there is an extra factor $\sim J$ when we estimate the difference in (3.27) since $\left|a^{2 J+1}-b^{2 J+1}\right| \lesssim$ $\left(\sum_{j=1}^{2 J+1} a^{2 J+1-j} b^{j-1}\right)|a-b|$ has $O(J)$ many terms. However, this does not cause a problem since the constant we obtain decays like a fractional power of a factorial in $J$ (as we see below in (3.29).) The same comment applies to Lemmata 3.12 and 3.13 .

As in the proof of Lemma 3.8, for fixed $n^{(j)}$ and $\mu_{j}$, there are at most $o\left(\left|\mu_{j}\right|^{0+}\right)$ many choices for $n_{1}^{(j)}, n_{2}^{(j)}$, and $n_{3}^{(j)}$. Also, note that $\mu_{j}$ is determined by $\widetilde{\mu}_{1}, \ldots, \widetilde{\mu}_{j}$ and

$$
\left|\mu_{j}\right| \lesssim \max \left(\left|\widetilde{\mu}_{j-1}\right|,\left|\widetilde{\mu}_{j}\right|\right)
$$

\footnotetext{
${ }^{9}$ The implicit constants are independent of $J$. The same comment applies to Lemmata 3.12 and 3.13.
} 
since $\mu_{j}=\widetilde{\mu}_{j}-\widetilde{\mu}_{j-1}$. Then, by Cauchy-Schwarz inequality, we have

$$
\begin{aligned}
\left\|\mathcal{N}_{0}^{(J+1)}(v)\right\|_{L^{2}} \lesssim \sum_{\mathcal{T}_{J} \in \mathfrak{T}(J)}\left\{\sum_{n}\left(\sum_{\substack{\left|\mu_{1}\right|>N \\
\left|\widetilde{\mu}_{j}\right|>(2 j+1)^{3} N^{1-\frac{1}{100}} \\
j=2, \ldots, J}} \prod_{k=1}^{J} \frac{1}{\left|\widetilde{\mu}_{k}\right|^{2}}\left|\mu_{k}\right|^{0+}\right)\right. \\
\left.\qquad\left(\sum_{\substack{\mathbf{n} \in \mathfrak{N}\left(\mathcal{T}_{J}\right) \\
\mathbf{n}_{r}=n}} \prod_{a \in \mathcal{T}_{J}^{\infty}}\left|v_{n_{a}}\right|^{2}\right)\right\}^{\frac{1}{2}} \\
\lesssim \frac{c_{J}}{\prod_{j=2}^{J}(2 j+1)^{\frac{3}{2}-}} N^{-\frac{J}{2}+\frac{(J-1)}{200}+\|v\|_{L^{2}}^{2 J+1}} \\
\lesssim N^{-\frac{J}{2}+\frac{(J-1)}{200}+\|v\|_{L^{2}}^{2 J+1}}
\end{aligned}
$$

where $c_{J}=|\mathfrak{T}(J)|$ is defined in (3.2).

Lemma 3.12. Let $\mathcal{N}_{r}^{(J+1)}$ be as in (3.24). Then, we have

$$
\begin{aligned}
&\left\|\mathcal{N}_{r}^{(J+1)}(v)\right\|_{L^{2}} \lesssim N^{-\frac{J}{2}+\frac{(J-1)}{200}+}\|v\|_{L^{2}}^{2 J+3}, \\
&\left.\left\|\mathcal{N}_{r}^{(J+1)}(v)-\mathcal{N}_{r}^{(J+1)}(w)\right\|_{L^{2}} \lesssim N^{-\frac{J}{2}+\frac{(J-1)}{200}+}+\|v\|_{L^{2}}^{2 J+2}+\|w\|_{L^{2}}^{2 J+2}\right)\|v-w\|_{L^{2}} .
\end{aligned}
$$

Proof. This lemma follows from Lemmata 3.11 and 2.1. Note that, given $\mathcal{T}_{J} \in \mathfrak{T}(J)$, we have $\#\left\{b: b \in \mathcal{T}_{J}^{\infty}\right\}=2 J+1$. This extra factor $2 J+1$ does not cause a problem thanks to the fast decaying constant in (3.29).

Finally, we treat $\mathcal{N}^{(J+1)}$. As before, we write

$$
\mathcal{N}^{(J+1)}=\mathcal{N}_{1}^{(J+1)}+\mathcal{N}_{2}^{(J+1)},
$$

where $\mathcal{N}_{1}^{(J+1)}$ is the restriction of $\mathcal{N}^{(J+1)}$ onto

$$
C_{J}=\left\{\left|\widetilde{\mu}_{J+1}\right| \lesssim(2 J+3)^{3}\left|\widetilde{\mu}_{J}\right|^{1-\frac{1}{100}}\right\} \cup\left\{\left|\widetilde{\mu}_{J+1}\right| \lesssim(2 J+3)^{3}\left|\mu_{1}\right|^{1-\frac{1}{100}}\right\}
$$

and $\mathcal{N}_{2}^{(J+1)}:=\mathcal{N}^{(J+1)}-\mathcal{N}_{1}^{(J+1)}$. We estimate the first term $\mathcal{N}_{1}^{(J+1)}$ in the following lemma, while we apply Poincaré-Dulac normal form reduction once again to the second term $\mathcal{N}_{2}^{(J+1)}$ as in (3.24).

Lemma 3.13. Let $\mathcal{N}_{1}^{(J+1)}$ be as in (3.32). Then, we have

$$
\begin{aligned}
&\left\|\mathcal{N}_{1}^{(J+1)}(v)\right\|_{L^{2}} \lesssim N^{-\frac{J-1}{2}+\frac{(J-2)}{200}+\|v\|_{L^{2}}^{2 J+3}}, \\
&\left\|\mathcal{N}_{1}^{(J+1)}(v)-\mathcal{N}_{1}^{(J+1)}(w)\right\|_{L^{2}} \lesssim N^{-\frac{J-1}{2}+\frac{(J-2)}{200}+}\left(\|v\|_{L^{2}}^{2 J+2}+\|w\|_{L^{2}}^{2 J+2}\right)\|v-w\|_{L^{2}} .
\end{aligned}
$$

Proof. We only prove (3.34) since (3.35) follows in a similar manner. As before, we use the divisor counting argument. For fixed $n^{(j)}$ and $\mu_{j}$, there are at most $o\left(\left|\mu_{j}\right|^{0+}\right)$ many choices for $n_{1}^{(j)}, n_{2}^{(j)}$, and $n_{3}^{(j)}$. Also, note that $\mu_{j}$ is determined by $\widetilde{\mu}_{1}, \ldots, \widetilde{\mu}_{j}$

First, we assume that $\left|\widetilde{\mu}_{J+1}\right|=\left|\widetilde{\mu}_{J}+\mu_{J+1}\right| \lesssim(2 J+3)^{3}\left|\widetilde{\mu}_{J}\right|^{1-\frac{1}{100}}$ holds in (3.33). Then, we have $\left|\mu_{J+1}\right| \lesssim\left|\widetilde{\mu}_{J}\right|$. Also, for fixed $\widetilde{\mu}_{J}$, there are at most $o\left(\left|\widetilde{\mu}_{J}\right|^{1-\frac{1}{100}}\right)$ many choices $\sqrt{10}$ for

\footnotetext{
${ }^{10}$ Strictly speaking, there are at most $o\left((2 J+3)^{3}\left|\widetilde{\mu}_{J}\right|^{1-\frac{1}{100}}\right)$ choices. However, we drop $(2 J+3)^{3}$ in view of fast decay of coefficients in $J$. See (3.29). The same comment applies in the following.
} 
$\widetilde{\mu}_{J+1}$ and hence for $\mu_{J+1}=\widetilde{\mu}_{J+1}-\widetilde{\mu}_{J}$. Then, by Cauchy-Schwarz inequality with (3.25) and (3.28), we have

$$
\begin{aligned}
& \left\|\mathcal{N}_{1}^{(J+1)}(v)\right\|_{L^{2}} \lesssim \sum_{\mathcal{T}_{J+1} \in \mathfrak{T}(J+1)}\left\{\sum_{n}\left(\sum_{\left|\mu_{1}\right|>N}\left|\widetilde{\mu}_{J}\right|^{1-\frac{1}{100}+} \prod_{k=1}^{J} \frac{1}{\left|\widetilde{\mu}_{k}\right|^{2}}\left|\mu_{k}\right|^{0+}\right)\right.
\end{aligned}
$$

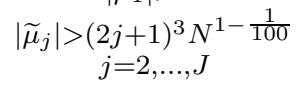

$$
\begin{aligned}
& \times\left(\left.\sum_{\substack{\mathbf{n} \in \mathfrak{N}\left(\mathcal{T}_{J+1}\right) \\
\mathbf{n}_{r}=n}} \prod_{\substack{a \in \mathcal{T}_{J+1}^{\infty} \\
\mid v_{n_{a}}}}\right|^{2}\right\}^{\frac{1}{2}} \\
& \lesssim N^{-\frac{J-1}{2}+\frac{J-2}{200}-\frac{1}{200}+}\|v\|_{L^{2}}^{2 J+3} \leq N^{-\frac{J-1}{2}+\frac{J-2}{200}+}\|v\|_{L^{2}}^{2 J+3} .
\end{aligned}
$$

by crudely estimating in $N$.

If $\left|\widetilde{\mu}_{J+1}\right| \lesssim(2 J+3)^{3}\left|\mu_{1}\right|^{1-\frac{1}{100}}$ holds in (3.33), then, for fixed $\mu_{j}, j=1, \ldots, J$, there are at most $O\left(\left|\mu_{1}\right|^{1-\frac{1}{100}}\right)$ many choices for $\mu_{J+1}$. By repeating the same computation, we obtain

$$
\left|\mu_{1}\right|^{1-\frac{1}{100}} \prod_{k=1}^{J} \frac{1}{\left|\widetilde{\mu}_{k}\right|^{2}}\left|\mu_{k}\right|^{0+}
$$

in (3.36), yielding (3.34) with $N^{-\frac{J-1}{2}+\frac{J-2}{200}+}$.

\section{EXISTENCE OF WEAK SOLUTIONS}

In this section, we put together all the lemmata in the previous sections and prove Theorems 1.4 in $L^{2}(\mathbb{T})$, i.e. for $s=0$. The argument for $s>0$ follows in a similar manner and we omit the details. By performing an infinite iteration of Poincaré-Dulac normal form reductions described in Sections 2 and 3, we have the following.

First consider a smooth solution $v$ of (2.1) with smooth initial condition $v_{0}$. Then, it satisfies the Duhamel formulation:

$$
\begin{aligned}
v(t) & =v_{0}+i \int_{0}^{t} \mathcal{N}_{1}(v)\left(t^{\prime}\right)-\mathcal{R}_{1}(v)\left(t^{\prime}\right)+\mathcal{R}_{2}\left(t^{\prime}\right) d t^{\prime} \\
& =v_{0}+i \int_{0}^{t} S\left(-t^{\prime}\right)\left[S\left(t^{\prime}\right) v\left(t^{\prime}\right)\left|S\left(t^{\prime}\right) v\left(t^{\prime}\right)\right|^{2}\right] d t^{\prime}
\end{aligned}
$$

as a smooth function for each $t$. Then it formally satisfies 11

$$
\partial_{t} v=\partial_{t} \sum_{j=2}^{\infty} \mathcal{N}_{0}^{(j)}(v)+\mathcal{R}_{1}+\mathcal{R}_{2}+\sum_{j=2}^{\infty} \mathcal{N}_{r}^{(j)}(v)+\sum_{j=1}^{\infty} \mathcal{N}_{1}^{(j)}(v)
$$

or

$$
\begin{aligned}
v(t)=\Gamma_{v_{0}} v(t):=v_{0} & +\sum_{j=2}^{\infty} \mathcal{N}_{0}^{(j)}(v)(t)-\sum_{j=2}^{\infty} \mathcal{N}_{0}^{(j)}\left(v_{0}\right) \\
& +\int_{0}^{t} \mathcal{R}_{1}\left(t^{\prime}\right)+\mathcal{R}_{2}\left(t^{\prime}\right)+\sum_{j=2}^{\infty} \mathcal{N}_{r}^{(j)}(v)\left(t^{\prime}\right)+\sum_{j=1}^{\infty} \mathcal{N}_{1}^{(j)}(v)\left(t^{\prime}\right) d t^{\prime},
\end{aligned}
$$

\footnotetext{
${ }^{11}$ Once again, we are replacing \pm 1 and $\pm i$ by 1 for simplicity since they play no role in our analysis.
} 
where $\mathcal{N}_{1}^{(1)}=\mathcal{N}_{11}$ in (2.4), $\mathcal{N}_{0}^{(2)}=\mathcal{N}_{21}$ in (2.10), $\mathcal{N}^{(2)}=\mathcal{N}_{3}$ in (3.5), and $\mathcal{N}_{r}^{(2)}=\mathcal{N}_{4}$ in (2.14). At this point, the right hand sides of (4.2) and (4.3) are merely formal expressions. In the following, we show that the series appearing on the right hand side of (4.3) converge absolutely in $C\left([0, T] ; L^{2}\right)$ for sufficiently small $T>0$ if $v \in C\left([0, T] ; L^{2}\right)$.

First, define the partial sum operator $\Gamma_{v_{0}}^{(J)}$ by

$$
\begin{aligned}
\Gamma_{v_{0}}^{(J)} v(t)=v_{0}+\sum_{j=2}^{J} & \mathcal{N}_{0}^{(j)}(v)(t)-\sum_{j=2}^{J} \mathcal{N}_{0}^{(j)}\left(v_{0}\right) \\
& +\int_{0}^{t} \mathcal{R}_{1}\left(t^{\prime}\right)+\mathcal{R}_{2}\left(t^{\prime}\right)+\sum_{j=2}^{J} \mathcal{N}_{r}^{(j)}(v)\left(t^{\prime}\right)+\sum_{j=1}^{J} \mathcal{N}_{1}^{(j)}(v)\left(t^{\prime}\right) d t^{\prime} .
\end{aligned}
$$

In the following, we let $C_{T} L^{2}=C\left([0, T] ; L^{2}\right)$. By Lemmata 2.1, 2.2, 3.11, 3.12, and 3.13, we have

$$
\begin{aligned}
\left\|\Gamma_{v_{0}}^{(J)} v\right\|_{C_{T} L^{2}} \leq\left\|v_{0}\right\|_{L^{2}} & +C \sum_{j=2}^{J} N^{-\frac{j-1}{2}+\frac{j-2}{200}+}\left(\|v\|_{C_{T} L^{2}}^{2 j-1}+\left\|v_{0}\right\|_{L^{2}}^{2 j-1}\right) \\
+C T & \left\{\|v\|_{C_{T} L^{2}}^{3}+\sum_{j=2}^{J} N^{-\frac{j-1}{2}+\frac{j-2}{200}+}\|v\|_{C_{T} L^{2}}^{2 j+1}\right. \\
& \left.+N^{\frac{1}{2}+}\|v\|_{C_{T} L^{2}}^{3}+\sum_{j=2}^{J} N^{-\frac{j-2}{2}+\frac{j-3}{200}+}\|v\|_{C_{T} L^{2}}^{2 j+1}\right\} .
\end{aligned}
$$

Suppose that $\left\|v_{0}\right\|_{L^{2}} \leq R$ and $\|v\|_{C_{T} L^{2}} \leq \widetilde{R}$ with $\widetilde{R} \geq R \geq 1$. Then, we have

$$
\begin{aligned}
& \left\|\Gamma_{v_{0}}^{(J)} v\right\|_{C_{T} L^{2}} \leq R+C N^{-\frac{1}{2}+} R^{3} \sum_{j=0}^{J-2}\left(N^{-\frac{1}{2}+\frac{1}{200}+} R^{2}\right)^{j}+C N^{-\frac{1}{2}+} \widetilde{R}^{3} \sum_{j=0}^{J-2}\left(N^{-\frac{1}{2}+\frac{1}{200}+} \widetilde{R}^{2}\right)^{j} \\
& +C T\left\{\left(1+N^{\frac{1}{2}+}\right) \widetilde{R}^{3}+N^{-\frac{1}{2}+} \widetilde{R}^{5} \sum_{j=0}^{J-2}\left(N^{-\frac{1}{2}+\frac{1}{200}+} \widetilde{R}^{2}\right)^{j}\right. \\
& \left.+N^{-\frac{1}{200+}} \widetilde{R}^{5} \sum_{j=0}^{J-2}\left(N^{-\frac{1}{2}+\frac{1}{200}+} \widetilde{R}^{2}\right)^{j}\right\} .
\end{aligned}
$$

Now, choose $N=N(\widetilde{R})$ large such that $N^{-\frac{1}{2}+\frac{1}{200}+} \widetilde{R}^{2} \leq \frac{1}{2}$. For example, we can simply choose

$$
N^{-\frac{1}{3}} \widetilde{R}^{2} \leq \frac{1}{2} \quad \Longleftrightarrow \quad N \geq\left(2 \widetilde{R}^{2}\right)^{3}
$$

Then, the geometric series in (4.6) converge (even for $J=\infty$ ) and are bounded by 2. Thus, we have

$$
\begin{aligned}
\left\|\Gamma_{v_{0}}^{(J)} v\right\|_{C_{T} L^{2}} \leq R+ & 2 C N^{-\frac{1}{2}+} R^{3}+2 C N^{-\frac{1}{2}+} \widetilde{R}^{3} \\
& +C T\left\{\left(1+N^{\frac{1}{2}+}\right) \widetilde{R}^{2}+2 N^{-\frac{1}{2}+} \widetilde{R}^{4}+2 N^{-\frac{1}{200+}} \widetilde{R}^{4}\right\} \widetilde{R} .
\end{aligned}
$$

Next, choose $T>0$ sufficiently small such that

$$
C T\left\{\left(1+N^{\frac{1}{2}+}\right) \widetilde{R}^{2}+2 N^{-\frac{1}{2}+} \widetilde{R}^{4}+2 N^{-\frac{1}{200+}} \widetilde{R}^{4}\right\}<\frac{1}{10} .
$$


From (4.7), we have $2 C N^{-\frac{1}{2}+} \widetilde{R}^{3} \leq C N^{-\frac{1}{6}+} \widetilde{R}$. Finally, by further imposing $N$ sufficiently large such that

we obtain

$$
C N^{-\frac{1}{6}+}<\frac{1}{10}
$$

$$
\left\|\Gamma_{v_{0}}^{(J)} v(t)\right\|_{C_{T} L^{2}} \leq R+\frac{1}{10} R+\frac{1}{5} \widetilde{R}=\frac{11}{10} R+\frac{1}{5} \widetilde{R} .
$$

We point out that this estimate also holds for $J=\infty$, and hence $\Gamma_{v_{0}}=\Gamma_{v_{0}}^{(\infty)}$ (= right hand side of (4.3) ) is well-defined.

Next, given an initial condition $v_{0} \in L^{2}(\mathbb{T})$, we construct a solution $v \in C\left([0, T] ; L^{2}\right)$ in the sense of Definition 1.3. First, take a sequence $\left\{v_{0}^{[m]}\right\}_{m \in \mathbb{N}}$ of smooth functions such that $v_{0}^{[m]} \rightarrow v_{0}$ in $L^{2}(\mathbb{T})$. (Simply take $v_{0}^{[m]}:=\mathbb{P}_{\leq m} v_{0}$, where $\mathbb{P}_{\leq m}$ is the Dirichlet projection onto the frequencies $|n| \leq m$.) Let $R=\left\|v_{0}\right\|_{L^{2}}+1$. Without loss of generality, assume that $\left\|v_{0}^{[m]}\right\|_{L^{2}} \leq R$.

In the following, we establish an a priori estimate on smooth solutions without the $L^{2}$ conservation so that the argument can be easily modified for $v_{0} \in H^{s}, s>0$. Let $v^{[m]}$ denote the smooth global-in-time solution of cubic NLS (2.1) with initial condition $v_{0}^{[m]}$. First, we use the continuity argument to show that $v^{[m]}$ satisfies (4.3) on $[0, T]$ with $T=T(R)>0$, independent of $m \in \mathbb{N}$. (As we see later, it suffices to take $T=T(R)>0$ satisfying (4.9).) Fix $m \in \mathbb{N}$. Note that $\left\|v^{[m]}\right\|_{C_{t} L^{2}}=\left\|v^{[m]}\right\|_{C\left([0, t] ; L^{2}\right)}$ is continuous in $t$. Since $\left\|v_{0}^{[m]}\right\|_{L^{2}} \leq R$, there exists a time interval $\left[0, T_{1}\right]$ with $T_{1}>0$ such that $\left\|v^{[m]}\right\|_{C_{T_{1}} L^{2}} \leq 4 R$. Then, by repeating the previous computation with $\widetilde{R}=4 R$ (and keeping one of the factors as $\left.\|v\|_{C_{T_{1}} L^{2}}\right)$, we obtain

$$
\left\|v^{[m]}\right\|_{C_{T_{1}} L^{2}}=\left\|\Gamma_{v_{0}^{[m]}} v^{[m]}\right\|_{C_{T_{1}} L^{2}} \leq \frac{11}{10} R+\frac{1}{5}\left\|v^{[m]}\right\|_{C_{T_{1}} L^{2}}
$$

as long as $N$ and $T_{1}$ satisfy (4.7), (4.9), and (4.10). This implies that

$$
\left\|v^{[m]}\right\|_{C_{T_{1}} L^{2}} \leq \frac{19}{10} R<2 R
$$

Hence, it follows from the continuity in $t$ of $\left\|v^{[m]}\right\|_{C_{t} L^{2}}$ that there exists $\varepsilon>0$ such that $\left\|v^{[m]}\right\|_{C_{T_{1}+\varepsilon} L^{2}} \leq 4 R$. Then, from (4.12) and (4.13) with $T_{1}+\varepsilon$ in place of $T_{1}$, we conclude that $\left\|v^{[m]}\right\|_{C_{T_{1}+\varepsilon} L^{2}} \leq 2 R$ as long as $N$ and $T_{1}+\varepsilon$ satisfy (4.7), (4.9), and (4.10). Note that these conditions are independent of $m \in \mathbb{N}$. In this way, we obtain a time interval $[0, T]$ such that $\left\|v^{[m]}\right\|_{C_{T} L^{2}} \leq 2 R$ for all $m \in \mathbb{N}$.

Moreover, by repeating a similar computation on the difference, we have

$$
\begin{aligned}
& \left\|\Gamma_{v_{0}^{\left[m_{1}\right]}} v^{\left[m_{1}\right]}-\Gamma_{v_{0}^{\left[m_{2}\right]}} v^{\left[m_{2}\right]}\right\|_{C_{T} L^{2}} \\
& \leq\left(1+\frac{1}{10}\right)\left\|v_{0}^{\left[m_{1}\right]}-v_{0}^{\left[m_{2}\right]}\right\|_{L^{2}}+\frac{1}{5}\left\|v^{\left[m_{1}\right]}-v^{\left[m_{2}\right]}\right\|_{C_{T} L^{2}}
\end{aligned}
$$

by possibly taking larger $N$ and smaller $T$. Since $v^{\left[m_{j}\right]}$ is a (smooth) solution with initial condition $v_{0}^{\left[m_{j}\right]}$, namely $v^{\left[m_{j}\right]}=\Gamma_{v_{0}^{\left[m_{j}\right]}} v^{\left[m_{j}\right]}$, it follows from (4.14) that

$$
\left\|v^{\left[m_{1}\right]}-v^{\left[m_{2}\right]}\right\|_{C_{T} L^{2}} \leq C^{\prime}\left\|v_{0}^{\left[m_{1}\right]}-v_{0}^{\left[m_{2}\right]}\right\|_{L^{2}}
$$

for some $C^{\prime}>0$. Hence, $\left\{v^{[m]}\right\}$ converges in $C\left([0, T] ; L^{2}\right)$.

Let $v^{\infty}$ denote the limit. Next, we show that $u^{\infty}:=S(t) v^{\infty}$ satisfies NLS (1.1) on $[0, T]$ in the sense of Definition 1.3. In the following, we drop $\infty$ in the superscript and simply 
denote $v^{\infty}$ and $u^{\infty}$ by $v$ and $u$. Also, let $u^{[m]}(t):=S(t) v^{[m]}(t)$, where $v^{[m]}$ is the smooth solution to (2.1) with smooth initial condition $v_{0}^{[m]}$ as above. Note that $u^{[m]}$ is the smooth solution to (1.1) with smooth initial condition $u_{0}^{[m]}:=v_{0}^{[m]}$. Moreover, $u^{[m]}$ converges to $u$ in $C\left([0, T] ; L^{2}\right)$, since $v^{[m]}$ converges to $v$ in $C\left([0, T] ; L^{2}\right)$. Thus, $\partial_{t} u^{[m]}$ and $\partial_{x}^{2} u^{[m]}$ converge to $\partial_{t} u$ and $\partial_{x}^{2} u$ in $\mathcal{D}^{\prime}(\mathbb{T} \times(0, T))$, respectively. Since $u^{[m]}$ satisfies (1.1) for each $m$, we see that

$$
\mathcal{N}\left(u^{[m]}\right):=u^{[m]}\left|u^{[m]}\right|^{2}=-i \partial_{t} u^{[m]}+\partial_{x}^{2} u^{[m]}
$$

also converges to some distribution $w$ in $\mathcal{D}^{\prime}(\mathbb{T} \times(0, T))$.

Proposition 4.1. Let $w$ be the limit of $\mathcal{N}\left(u^{[m]}\right)$ in the distributional sense as above. Then, $w=\mathcal{N}(u)$, where $\mathcal{N}(u)$ on the right hand side is to be interpreted in the sense of Definition 1.2.

We present the proof of Proposition 4.1 at the end of this section. Assuming Proposition 4.1, we see that $u=u^{\infty}$ is a solution to (1.1) in the extended sense as in Definition 1.3.

It follows from (4.7), (4.9), and (4.10) (with $R=\left\|v_{0}\right\|_{L^{2}}+1$ and $\widetilde{R}=4 R$ ) that the time of existence $T$ satisfies $T \gtrsim\left(1+\left\|v_{0}\right\|_{L^{2}}\right)^{-\beta}$ for some $\beta>0$. The Lipschitz dependence on initial data follows from (4.15), bypassing smooth approximations.

Lastly, for $s>0$, we only have to note that all the lemmata in Sections 2 and 3 hold true even if we replace the $L^{2}$-norm by the $H^{s}$-norm. Indeed, if $n^{(j)}$ is large, then there exists at least one of $n_{1}^{(j)}, n_{2}^{(j)}$, and $n_{3}^{(j)}$ satisfies $\left|n_{k}^{(j)}\right| \geq \frac{1}{3}\left|n^{(j)}\right|$, since we have $n^{(j)}=n_{1}^{(j)}-n_{2}^{(j)}+n_{3}^{(j)}$. Hence, in the estimates for the terms in the $J$ th generation (Lemmata 3.11, 3.12, 3.13), there exists at least one frequency $n_{k}^{(j)}$ (with some $j=1, \ldots, J$ ) such that

$$
\langle n\rangle^{s} \leq 3^{j s}\left\langle n_{k}^{(j)}\right\rangle^{s} \leq 3^{J s}\left\langle n_{k}^{(j)}\right\rangle^{s} .
$$

Note that the constant grows exponentially in $J$. However, this exponential growth does not cause a problem thanks to the factorial decay in the denominator (as seen in the proof Lemma 3.11.)

Proof of Proposition 4.1. Let $\left\{T_{N}\right\}_{N \in \mathbb{N}}$ be a sequence of Fourier cutoff multipliers as in Definition 1.1. Fix a test function on $\mathbb{T} \times(0, T)$. Then, we need to show that given $\varepsilon>0$, there exists $N_{0}$ such that for all $N \geq N_{0}$, we have

$$
\left|\left\langle w-\mathcal{N}\left(T_{N} u\right), \phi\right\rangle\right|<\varepsilon .
$$

Write the left hand side of (4.16) as

$$
\begin{aligned}
\left|\left\langle w-\mathcal{N}\left(T_{N} u\right), \phi\right\rangle\right| \leq\left|\left\langle w-\mathcal{N}\left(u^{[m]}\right), \phi\right\rangle\right| & +\left|\left\langle\mathcal{N}\left(u^{[m]}\right)-\mathcal{N}\left(T_{N} u^{[m]}\right), \phi\right\rangle\right| \\
& +\left|\left\langle\mathcal{N}\left(T_{N} u^{[m]}\right)-\mathcal{N}\left(T_{N} u\right), \phi\right\rangle\right| .
\end{aligned}
$$

By definition of of $w$, we see that

$$
\left|\left\langle w-\mathcal{N}\left(u^{[m]}\right), \phi\right\rangle\right|<\frac{1}{3} \varepsilon
$$

for sufficiently large $m \in \mathbb{N}$.

Next, consider the second term for fixed $m$. By writing $\mathcal{N}\left(u^{[m]}\right)-\mathcal{N}\left(T_{N} u^{[m]}\right)$ in a telescoping sum, we only consider

$$
\left|\left\langle\mathcal{N}\left(\left(I-T_{N_{1}}\right) u^{[m]}, u^{[m]}, u^{[m]}\right), \phi\right\rangle\right|,
$$


where $\mathcal{N}\left(u_{1}, u_{2}, u_{3}\right)=u_{1} \overline{u_{2}} u_{3}$ and $I$ denotes the identity operator. (The other terms in the telescoping sum have similar forms.) By Hölder inequality and Sobolev embedding, we obtain

$$
\begin{aligned}
\left|\left\langle\mathcal{N}\left(\left(I-T_{N_{1}}\right) u^{[m]}, u^{[m]}, u^{[m]}\right), \phi\right\rangle\right| \leq\|\phi\|_{L_{x, T}^{2}}\left\|u^{[m]}\right\|_{L_{x, T}^{\infty}}^{2}\left\|\left(I-T_{N}\right) u^{[m]}\right\|_{L_{x, T}^{2}} \\
\quad \leq C_{\phi}\left\|u^{[m]}\right\|_{C_{T} H^{\frac{1}{2}+}}^{2}\left\|\left(I-T_{N}\right) u^{[m]}\right\|_{L_{x, T}^{2}} \\
\leq C_{\phi, m}\left\|\left(I-T_{N}\right) u^{[m]}\right\|_{L_{x, T}^{2}},
\end{aligned}
$$

where $L_{x, T}^{2}$ denotes $L^{2}(\mathbb{T} \times[0, T])$. Here, we used the fact that $\left\|u^{[m]}\right\|_{C_{T} H^{\frac{1}{2}+}}^{2}$ is a finite constant (depending on $m$.) By definition of the Fourier cutoff operators, $\left(\left(I-T_{N}\right) u^{[m]}\right)^{\wedge}(n, t)$ converges pointwise in $n$ and $t$. Then, by Dominated Convergence Theorem, there exists $N_{0}=N_{0}(m)$ such that

$$
(4.18)<\frac{1}{3} \varepsilon
$$

for all $N \geq N_{0}$.

As for the third term, first consider the sequence $\left\{\mathcal{N}\left(T_{N} u^{[m]}\right)\right\}_{m \in \mathbb{N}}$ for each fixed $N$. By applying the Poincaré-Dulac normal form reduction to $\left\{S(-t) \mathcal{N}\left(T_{N} u^{[m]}\right)\right\}_{m \in \mathbb{N}}$ (which is basically the nonlinearity in the $v$-equation (2.1) modulo $T_{N}$ ) as in Sections 2 and 3 , we see that $\left\{\mathcal{N}\left(T_{N} u^{[m]}\right)\right\}_{m \in \mathbb{N}}$ is a Cauchy sequence in $\mathcal{D}^{\prime}(\mathbb{T} \times(0, T))$, as $m \rightarrow \infty$ for each fixed $N$ since $u^{[m]}$ is Cauchy in $C\left([0, T] ; L^{2}\right)$. Moreover, this convergence is uniform in $N$ since the multipliers for $T_{N}$ are uniformly bounded in $N$.

On the other hand, note that for fixed $N, T_{N} u$ is in $C_{T} H^{\infty}$, since the multiplier $m_{N}$ for $T_{N}$ has a compact support. Thus, $\mathcal{N}\left(T_{N} u\right)=T_{N} u\left|T_{N} u\right|^{2}$ makes sense as a function. Hence, for fixed $N$, we can choose $m$ large such that

$$
\begin{aligned}
\left|\left\langle\mathcal{N}\left(T_{N} u^{[m]}\right)-\mathcal{N}\left(T_{N} u\right), \phi\right\rangle\right| & \leq\|\phi\|_{L_{x, T}^{4}}\left(\left\|T_{N} u^{[m]}\right\|_{L_{x, T}^{4}}^{2}+\left\|T_{N} u\right\|_{L_{x, T}^{4}}^{2}\right)\left\|T_{N} u^{[m]}-T_{N} u\right\|_{L_{x, T}^{4}}^{2} \\
& \leq C_{\phi,\|u\|_{C_{T} L^{2}}} M^{\frac{3}{4}} T^{\frac{3}{4}}\left\|u^{[m]}-u\right\|_{C_{T} L^{2}}^{2}<\frac{1}{3} \varepsilon,
\end{aligned}
$$

by Sobolev inequality, where $M=M(N) \in \mathbb{N}$ is chosen such that $\operatorname{supp}\left(m_{N}\right) \subset[-M, M]$. i.e. $\mathcal{N}\left(T_{N} u^{[m]}\right)$ converges to $\mathcal{N}\left(T_{N} u\right)$ in $\mathcal{D}^{\prime}(\mathbb{T} \times(0, T))$ as $m \rightarrow \infty$ for each fixed $N$.

Combining these two observations, we conclude that $\mathcal{N}\left(T_{N} u^{[m]}\right)$ converges to $\mathcal{N}\left(T_{N} u\right)$ in $\mathcal{D}^{\prime}(\mathbb{T} \times(0, T))$ as $m \rightarrow \infty$ uniformly in $N$. Namely,

$$
\left|\left\langle\mathcal{N}\left(T_{N} u^{[m]}\right)-\mathcal{N}\left(T_{N} u\right), \phi\right\rangle\right|<\frac{1}{3} \varepsilon
$$

for all sufficiently large $m$, uniformly in $N$. Therefore, (4.16) follows by first choosing $m$ sufficiently large such that (4.17) and (4.20) hold, then choosing $N_{0}=N_{0}(m)$ such that (4.19) holds.

\section{UnCONDItional Uniqueness in $C_{t} H^{s}, s \geq \frac{1}{6}$}

In this section, we prove Theorem 1.6. More precisely, we justify the formal computations in Sections 2 and 3 on the additional regularity assumption. Then, the Lipschitz bound implies the uniqueness. In the following, we justify our computations, assuming that $u$ is a solution to (1.1) in $C\left([0, T] ; L^{3}(\mathbb{T})\right)$.

First, we make sense of the use of $\partial_{t} v_{n}(t)=e^{-i n^{2} t} \widehat{u}(n, t)$ in Sections 2 and 3 , Suppose that $u \in C\left([0, T] ; L^{3}(\mathbb{T})\right)$. Then, we have $u|u|^{2} \in C\left([0, T] ; L^{1}(\mathbb{T})\right)$, and hence $\mathbb{P}_{\leq M}\left(u|u|^{2}\right) \in$ $C\left([0, T] ; H^{\infty}(\mathbb{T})\right)$ for any $M \in \mathbb{N}$, where $\mathbb{P}_{\leq M}$ is the Dirichlet projection onto the frequencies 
$|n| \leq M$. This implies $\left(\partial_{x}^{2} \mathbb{P}_{\leq M} u\right)^{\wedge} \in C\left([0, T] ; H^{\infty}(\mathbb{T})\right)$. Hence, from the equation (1.1), we see that $\left(\mathbb{P}_{\leq M} \partial_{t} u\right)^{\wedge} \in C\left([0, T] ; H^{\infty}(\mathbb{T})\right)$. In particular, $\widehat{u}(n, \cdot)$ is a $C^{1}$-function in $t$.

In Sections 2 and 3, we switched the order of summation and the time differentiation. For example, see (2.10). This can be justified, also by assuming $u \in C\left([0, T] ; L^{3}(\mathbb{T})\right)$. First, we state a lemma.

Lemma 5.1. Let $\left\{f_{n}\right\}_{n \in \mathbb{N}}$ be a sequence in $\mathcal{D}_{t}^{\prime}$. Suppose that $\sum_{n} f_{n}$ converges (absolutely) in $\mathcal{D}_{t}^{\prime}$. Then, $\sum_{n} \partial_{t} f_{n}$ converges (absolutely) in $\mathcal{D}_{t}^{\prime}$ and $\partial_{t}\left(\sum_{n} f_{n}\right)=\sum_{n} \partial_{t} f_{n}$.

Proof. Recall that a sequence of distribution $g_{n}$ is said to converge to a distribution $g$ if, for all $\phi \in \mathcal{D}$, we have $\left\langle g_{n}, \phi\right\rangle \rightarrow\langle g, \phi\rangle$. Thus, we have

$$
\left\langle\sum_{n=1}^{\infty} f_{n}^{\prime}, \phi\right\rangle=\lim _{N \rightarrow \infty}\left\langle\sum_{n=1}^{N} f_{n}^{\prime}, \phi\right\rangle=\lim _{N \rightarrow \infty} \sum_{n=1}^{N}\left\langle f_{n}^{\prime}, \phi\right\rangle,
$$

if the right hand side exists. By the definition of a distributional derivative, we have

$$
\lim _{N \rightarrow \infty} \sum_{n=1}^{N}\left\langle f_{n}^{\prime}, \phi\right\rangle=-\lim _{N \rightarrow \infty} \sum_{n=1}^{N}\left\langle f_{n}, \phi^{\prime}\right\rangle,
$$

where the right hand side converges since $\sum_{n} f_{n}$ converges in $\mathcal{D}^{\prime}$. Hence, $\sum_{n} f_{n}^{\prime}$ converges in $\mathcal{D}^{\prime}$. The second claim follows once we note the following.

$$
-\lim _{N \rightarrow \infty} \sum_{n=1}^{N}\left\langle f_{n}, \phi^{\prime}\right\rangle=-\lim _{N \rightarrow \infty}\left\langle\sum_{n=1}^{N} f_{n}, \phi^{\prime}\right\rangle=-\left\langle\sum_{n=1}^{\infty} f_{n}, \phi^{\prime}\right\rangle=\left\langle\partial_{t}\left(\sum_{n=1}^{\infty} f_{n}\right), \phi\right\rangle,
$$

where the second equality follows from the definition of $\sum_{n} f_{n}$ as a distributional limit.

Now, we consider (2.10) for fixed $n$. Then, we want to apply Lemma 5.1 to a sequence

$$
\left\{a_{n, n_{1}, n_{2}}(t)\right\}:=\left\{\frac{e^{-i \Phi(\bar{n}) t}}{2\left(n-n_{1}\right)\left(n-n_{3}\right)} v_{n_{1}}(t) \bar{v}_{n_{2}}(t) v_{n_{3}}(t)\right\},
$$

where $n=n_{1}-n_{2}+n_{3}$ and $\left(n, n_{1}, n_{2}, n_{3}\right) \in A(n)^{c}$. (Here, $a_{n, n_{1}, n_{2}}$ depends on several indices with a restriction (i.e. on $\left.A_{N}(n)^{c}\right)$, but we can arrange them to be a sequence.)

By Lemma 2.3. $\sum_{A_{N}(n)^{c}} a_{n, n_{1}, n_{2}}$ converges absolutely and is bounded in $C([0, T]$ ) (for fixed $n$.) In particular, for each $n_{1}$ and $n_{2}, a_{n, n_{1}, n_{2}}$ is a distribution on $[0, T]$. By Lemma 5.1, we have

$$
\begin{aligned}
\partial_{t}\left[\sum_{A_{N}(n)^{c}} \frac{e^{-i \Phi(\bar{n}) t}}{-i \Phi(\bar{n})} v_{n_{1}} \bar{v}_{n_{2}} v_{n_{3}}\right]=\sum_{A_{N}(n)^{c}} \partial_{t}\left[\frac{e^{-i \Phi(\bar{n}) t}}{-i \Phi(\bar{n})} v_{n_{1}} \bar{v}_{n_{2}} v_{n_{3}}\right] \\
=\sum_{A_{N}(n)^{c}} \partial_{t}\left(\frac{e^{-i \Phi(\bar{n}) t}}{-i \Phi(\bar{n})}\right) v_{n_{1}} \bar{v}_{n_{2}} v_{n_{3}}+\sum_{A_{N}(n)^{c}} \frac{e^{-i \Phi(\bar{n}) t}}{-i \Phi(\bar{n})} \partial_{t}\left(v_{n_{1}} \bar{v}_{n_{2}} v_{n_{3}}\right) .
\end{aligned}
$$

In the second equality, we applied the product rule. It is in this step that we needed the additional regularity $u \in C\left([0, T] ; L^{3}\right)$ so that $v_{n}$ is continuously differentiable and the product rule is applicable. A similar argument justifies the exchange of the sum and the time differentiation in the $J$ th generation. We omit the details.

Indeed, if we assume that $u \in C\left([0, T] ; L^{3}\right)$, we can say more on this issue. First, note that from (2.1), we have $\partial_{t} v_{n}=e^{-i n^{2} t} \mathcal{N}(S(-t) v)_{n}=e^{-i n^{2} t} \mathcal{N}(u)_{n}$. Then,

$$
\left\|\partial_{t} v_{n}\right\|_{C_{T} \ell_{n}^{\infty}}=\left\|\mathcal{N}(u)_{n}\right\|_{C_{T} \ell_{n}^{\infty}} \leq\|\mathcal{N}(u)\|_{C_{T} L_{x}^{1}} \leq\|u\|_{C_{T} L_{x}^{3}}^{3} .
$$


Hence, $\partial_{t} v_{n} \in C\left([0, T] ; \ell_{n}^{\infty}\right)$. In the following, fix $n$. Then, by a variant of Lemma 2.3, the second term on the right hand side of (5.1) is estimated as

$$
\left|\sum_{A_{N}(n)^{c}} \frac{e^{-i \Phi(\bar{n}) t}}{-i \Phi(\bar{n})} \partial_{t}\left(v_{n_{1}} \bar{v}_{n_{2}} v_{n_{3}}\right)\right| \lesssim\left\|\partial_{t} v_{n}\right\|_{C_{T} \ell_{n}^{\infty}}\|v\|_{C_{T} L^{2}}^{2} \leq\|u\|_{C_{T} L^{3}}^{3}\|v\|_{L^{2}}^{2},
$$

where the convergence is absolute and uniform (in t.) The first term in (5.1) can be written as $e^{-i n^{2} t} \mathcal{N}(u)_{n}$ and thus also converges in view of (5.2). (Here, the convergence is not absolute, but uniform in $t$.) Therefore, we can simply switch the sum and the time differentiation (i.e. the first equality in (5.1)) in classical sense. The argument for the $J$ th generation is similar and we omit the details.

Lastly, the regularity $C\left([0, T] ; L^{3}\right)$ was sufficient to justify the formal computations in Sections 2 and 3. However, in order to prove the lemmata, which are proven on the Fourier side, we need a $L^{2}$-based space of the same scaling, namely $C\left([0, T] ; H^{\frac{1}{6}}\right)$.

Acknowledgments: The authors would like to thank Prof. Nicolas Burq, Prof. Herbert Koch, and Prof. Nader Masmoudi for the helpful comments and discussions.

\section{REFERENCES}

[1] V.I. Arnold, Geometrical methods in the theory of ordinary differential equations, Second edition. Grundlehren der Mathematischen Wissenschaften [Fundamental Principles of Mathematical Sciences], 250. Springer-Verlag, New York, 1988. xiv+351 pp.

[2] A. Babin, A. Ilyin, E. Titi, On the regularization mechanism for the periodic Korteweg-de Vries equation, Comm. Pure Appl. Math. 64 (2011), no. 5, 591-648.

[3] J. Bourgain, Fourier transform restriction phenomena for certain lattice subsets and applications to nonlinear evolution equations I. Schrödinger equations, Geom. Funct. Anal. 3 (1993), no. 2, 107-156.

[4] J. Bourgain, Remarks on stability and diffusion in high-dimensional Hamiltonian systems and partial differential equations, Ergodic Theory Dynam. Systems 24 (2004), no. 5, 1331-1357.

[5] J. Bourgain, A remark on normal forms and the "I-method" for periodic NLS, J. Anal. Math. 94 (2004), $125-157$.

[6] N. Burq, P. Gérard, N. Tzvetkov, An instability property of the nonlinear Schrodinger equation on $\mathbb{S}^{d}$, Math. Res. Lett. 9 (2002), no. 2-3, 323-335.

[7] M. Christ, Nonuniqueness of weak solutions of the nonlinear Schrödinger equation, arXiv:math/ 0503366v1 [math.AP].

[8] M. Christ, Power series solution of a nonlinear Schrödinger equation, Mathematical aspects of nonlinear dispersive equations, 131-155, Ann. of Math. Stud., 163, Princeton Univ. Press, Princeton, NJ, 2007.

[9] M. Christ, J. Colliander, T. Tao, Asymptotics, frequency modulation, and low regularity ill-posedness for canonical defocusing equations, Amer. J. Math. 125 (2003), no. 6, 1235-1293.

[10] M. Christ, J. Colliander, T. Tao, Instability of the Periodic Nonlinear Schrödinger Equation, arXiv:math/0311227v1 [math.AP].

[11] J. Colliander, S. Kwon, T. Oh, A remark on normal forms and the "upside-down" I-method for periodic NLS: growth of higher Sobolev norms, arXiv:1010.2501v1 [math.AP].

[12] J. Colliander, T. Oh, Almost sure well-posedness of the periodic cubic nonlinear Schrödinger equation below $L^{2}(\mathbb{T})$, to appear in Duke Math. J.

[13] G. Furioli, F. Planchon, E. Terraneo, Unconditional well-posedness for semilinear Schrödinger and wave equations in $H^{s}$, Harmonic analysis at Mount Holyoke (South Hadley, MA, 2001), 147-156, Contemp. Math., 320, Amer. Math. Soc., Providence, RI, 2003.

[14] P. Germain, N. Masmoudi, J. Shatah, Global solutions for $3 D$ quadratic Schrödinger equations, Int. Math. Res. Not. 2009, no. 3, 414-432.

[15] J. Ginibre, An introduction to nonlinear Schrödinger equations, Nonlinear waves (Sapporo, 1995), 85133, GAKUTO Internat. Ser. Math. Sci. Appl., 10, Gakkōtosho, Tokyo, 1997.

[16] A. Grünrock, S. Herr, Low regularity local well-posedness of the derivative nonlinear Schrödinger equation with periodic initial data, SIAM J. Math. Anal. 39 (2008), no. 6, 1890-1920. 
[17] G.H. Hardy, E.M. Wright, An introduction to the theory of numbers, Fifth edition. The Clarendon Press, Oxford University Press, New York, 1979. xvi+426 pp.

[18] L. Molinet, On ill-posedness for the one-dimensional periodic cubic Schrödinger equation, Math. Res. Lett. 16 (2009), no. 1, 111-120.

[19] T. Kato, On nonlinear Schrödinger equations. II. $H^{s}$-solutions and unconditional well-posedness, J. Anal. Math. 67 (1995), 281-306.

[20] S. Kwon, T. Oh, On unconditional well-posedness of modified KdV, Internat. Math. Res. Not. (2011), doi: 10.1093/imrn/rnr156.

[21] J. Shatah, Normal forms and quadratic nonlinear Klein-Gordon equations, Comm. Pure Appl. Math. 38 (1985), no. 5, 685-696.

[22] H. Takaoka, Y. Tsutsumi, Well-posedness of the Cauchy problem for the modified KdV equation with periodic boundary condition, Int. Math. Res. Not. 2004, no. 56, 3009-3040.

[23] Y. Zhou, Uniqueness of weak solution of the KdV equation, Int. Math. Res. Not. 1997, no. 6, 271-283.

Zihua Guo, School of Mathematics, Institute for Advanced Study, Einstein Drive, PrinceTON, NJ 08540, USA

E-mail address: guo@math.ias.edu

Soonsik Kwon, Department of Mathematical Sciences, Korea Advanced Institute of Science and Technology, 335 Gwahangno, Yuseong-gu, Daejeon 305-701, Republic of Korea

E-mail address: soonsikk@kaist.edu

Tadahiro Oh, Department of Mathematics, Princeton University, Fine Hall, Washington RD., Princeton, NJ 08544-1000, USA

E-mail address: hirooh@math.princeton.edu 